Notre Dame Journal of Formal Logic

Volume 37, Number 4, Fall 1996

\title{
Logic in Russell's Principles of Mathematics
}

\author{
GREGORY LANDINI
}

\begin{abstract}
Unaware of Frege's 1879 Begriffsschrift, Russell's 1903 The Principles of Mathematics set out a calculus for logic whose foundation was the doctrine that any such calculus must adopt only one style of variablesentity (individual) variables. The idea was that logic is a universal and allencompassing science, applying alike to whatever there is-propositions, universals, classes, concrete particulars. Unfortunately, Russell's early calculus has appeared archaic if not completely obscure. This paper is an attempt to recover the formal system, showing its philosophical background and its semantic completeness with respect to the tautologies of a modern sentential calculus.
\end{abstract}

1 Introduction It is commonplace today to speak as though there are different "logics," for (after all) there are different deductive calculi. On this conception, "a logic" is just a formal deductive system and the study of logic is the study of uninterpreted calculi, their semantic completeness, consistency, decidability, and so on. This makes logic a branch of mathematics. Russell's conception of logic was quite different. Logic is not to be identified with any formal uninterpreted calculus and the science of logic must not be thought to be the theory of formal systems. According to Principles, logic is a synthetic a priori science that applies to every entity whatsoever. It is completely general and all-encompassing, and its subject matter is the structural forms of propositions grounding the relation of implication ([10, p. 457).

Russell (as Frege before him) spoke of himself as offering not merely a calculus ratiocinator in the manner of Boole, but a characteristica lingua universalis as Leibniz had conceived of it. ${ }^{1}$ In an important paper, van Heijenoort [15] interpreted Frege (and Russell) to be advocating a "syntactic approach" to logic according to which logic is a universal language with content in its own right, and logical truth and validity are to be construed in terms of deductive closure under the axioms. He contrasted this conception with the "semantic" approach of Tarski (originating in the algebraic tradition of Boole, Peirce, Schröder and Löweheim). Here logic is the study of uninterpreted deductive calculi-calculi which can express a content (e.g., mathematics) only when an interpretation over an appropriate domain (e.g., numbers) is

Received May 16, 1995; revised September 24, 1996 
provided. On the semantic view, logical truth and validity are construed in terms of invariant truth throughout all admissible interpretations. Van Heijenoort goes on to maintain that the Frege/Russell tradition excludes a distinction between the logical system and the metasystem required to justify and study the system. Accordingly, he went so far as to say that the Frege/Russell tradition actually obstructs metasystemic research (16], p. 116).

In van Heijenoort's view, Frege and Russell's conception of logic excludes the very intelligibility of now rather commonplace distinctions between syntax and semantics, object-language and meta-language, schemata and axioms, axioms and transformation rules, and so on. His view has been influential. In a recent book, Hylton agrees:

If logic is to be unconditionally and unrestrictedly true in the sense that Russell requires it to be, then it must be universally applicable. This, in turn, implies that statements about logic must themselves fall within the scope of logic, so the notion of a meta- theoretical perspective falls away. If this were not so, if logic were thought of as set up within a more inclusive meta-language, then ...it would appear that logic is not absolutely and unconditionally true. Logic, on this modern picture, is not unrestricted, for it is set up in a more inclusive language which must fall outside of its scope. (5], p. 203)

On the "logic as language" conception, logic must "contain its own meta-theory" in a way that makes such distinctions beyond the pale for Russell.

Now van Heijenoort is correct that for Russell, logical truth is not invariant truth in every admissible interpretation. Indeed, some of the formulas Russell counted as expressing logical truths are not invariantly true in every interpretation of the nonlogical words. For instance,

If $x$ and $y$ are any entities and $p$ is any proposition containing $x$ then there is a proposition exactly like $p$ but containing $y$ wherever $p$ contains $x$.

There is also,

$$
x \supset y \cdot \supset_{x, y} \cdot x \supset x
$$

which says that $x$ 's implying $y$ implies, for all $x$ and $y, x$ 's implying $x$. These are logical truths about propositions. Logic, in Russell's early view, is a synthetic a priori science of the structures of propositions. Logical truth is closure under the axioms, as van Heijenoort says; and indeed, any true fully closed formula of Russell's system will count as a logical truth. But van Heijenoort goes too far. None of this means that Russell's conception of logic requires that a system for logic includes its own meta-theory.

Frege made clear in his review of Peano that his begriffsschrift is both a lingua characteristica and a calculus ratiocinator, while Boolean algebra amounts at most to the latter (Frege [4], p. 7). The superiority Frege found in his begriffsschrift is not that it is itself a language with content as opposed to an uninterpreted syntax with rules for the manipulation of the symbols. The notion of content Frege speaks of as being part of his begriffsschrift and absent from Boole's is not one of semantic content given via descriptive constants of the language (predicate constants). The notion of content Frege has in mind is that of syntactic structure. The superiority of Frege's begriffsschrift lies in its comprehension principle for functions- that is, in its ability 
to decompose complex sentential contents (logical structures) in different ways-so that now one function and now another is singled out. Consider for instance,

Some object is left of George.

The logical content here is represented by the syntactic structure,

$$
\top^{x_{\top} f x a}
$$

of Frege's begriffsschrift. The content (structure) that this syntax represents (on its intended interpretation) permits decomposition in various ways. One way is

$$
f(a)
$$

where we have ' $a$ ' assigned to George and ' $f$ ' assigned to the following truthfunction,

$$
f x=\left\{\begin{array}{l}
\text { the True, if some object is left of } x \\
\text { the False, otherwise. }
\end{array}\right.
$$

Another way is to present this as the mutual saturation of two functions,

$$
\begin{aligned}
{ }_{\top} \beta_{\top} \mathrm{M} \beta & =\left\{\begin{array}{l}
\text { the True, if some object } \beta \text { is such that } \ldots, \beta, \ldots \\
\text { the False, otherwise. }
\end{array}\right. \\
h z & =\left\{\begin{array}{l}
\text { the True, if } z \text { is left of George } \\
\text { the False, otherwise. }
\end{array}\right.
\end{aligned}
$$

It is the availability of such decompositions that makes it possible to find logical structure in contents such as 'There are twelve apostles', and go on to uncover complex structure in such contents as ' $12>6$ ', contents that Kant's Transcendental Aesthetic had presumed to be simple. It is precisely the ability to express such structures that, in Frege's view, reveals that no nonlogical and uniquely mathematical intuitions of number are needed to capture the derivations of arithmetic. The same is true of Russell, although he did not devise a function calculus as Frege had.

It is important, then, to realize that Frege and Russell viewed logic as neither a formal uninterpreted calculus nor as a language "containing its own meta-theory." No mathematical realist would confuse the science of arithmetic with an axiom system of formal number theory. And similarly, one must not confuse the science of logic with the formal system - the lingua characterista universalis - which is proffered to capture it. In Russell's conception, logic is the science of the structure of propositions, in precisely that way the mathematical realist takes arithmetic to be the science of number. Seeing this, we realize that nothing in Russell's (or Frege's) conception of logic precludes the adoption of commonplace modern distinctions between theory and meta-theory. These distinctions are untendentious; they do not distinguish the modern mathematical conception of logic from the Frege/Russell conception.

But, it might be objected, if Russell and (Frege) viewed a lingua characteristica as a calculus in the modern sense, won't logic fail to be all-encompassing. If logic is universal and all-encompassing, so the argument goes, then it applies to all forms of reasoning, and accordingly meta-linguistic proofs and notions cannot have any special status. The whole meta-theoretical perspective collapses for else there would be 
an essentially richer and more inclusive realm of reasoning (reasoning in the metalanguage) which would have to fall outside the scope of logic.

The argument is wanting. To be sure, Russell's conception takes logic to be all-encompassing, for any reasoning, in virtue of being proper reasoning, uses logic. Logic encompasses all proper patterns of reasoning (and shows why others are improper). If a given formal calculus for logic fully captures logic-so that every relation is expressible and every function is representable in the ideography of an applied form of that calculus - then all proper reasoning patterns can be expressed within it. But suppose it is discovered ${ }^{2}$ that for any given calculus for logic, there will be patterns of reasoning (and notions pertaining to the relationship between the expressions of that calculus and their referents) that must lie outside the calculus. What of it? If it happens that some properties such as 'designates-in-s' and 'true-in-s' cannot be expressed in the language of a proposed calculus $s$ for logic, ${ }^{3}$ the discovery in no way impugns the claim that logic is all-encompassing.

But again, one might object that if we have a calculus in the modern sense then the variables of such a calculus (as with any calculus in the modern sense) can be interpreted over restricted domains. Moreover, it will be possible to alter the interpretation of the logical particles themselves. So be it! Russell was well aware that once a science is represented by an axiomatized calculus there can be many interpretations. He quite clearly observes that arithmetic can be taken to be about all progressions, for all satisfy the Peano/Dedekind Postulates under different interpretations of 'zero', 'number', and 'successor' ([10], p. 430). Russell did not try to avoid this by claiming that no formal calculus for arithmetic is possible. ${ }^{4}$ His response was that a logical (conceptual) analysis of the notion of 'natural number' reveals the Frege/Russell cardinals. In the same way, a conceptual analysis of the notion of 'continuity' and 'limit' had (in the hands of Weierstrass, Dedekind, et al.) yielded that it is order (relation) not magnitude (quantity) that is basic. That there may be unintended interpretations of any formal system intended to represent the structures revealed by the analysis of 'number', is simply irrelevant.

What then of Russell's famous doctrine of the unrestricted variable? In Principles, Russell held that logic applies to whatever has ontological status, irrespective of the sort of entity (concrete particular, universal, proposition, etc.) that it is. Russell maintains that unrestricted variables are required in any calculus for logic because any restricted variable would simply be in the antecedent clause of a conditional whose variables are unrestricted, or if themselves restricted, would in turn require a new antecedent clause delimiting the restriction. Eventually, one arrives at an unrestricted variable. ${ }^{5}$ Does this not show that Russell is trying to banish the very idea of an unintended interpretation by viewing logic as coming with a ready-made semantics?

Hylton [5] reads Russell this way. He argues that Russell's very argument for unrestricted variables precludes a distinction between object-language and metalanguage. It assumes from the onset that "...the statement which establishes the universe of discourse is on the same level as the assertion which is made once the discourse is established. Thus the former can be taken as antecedent and the latter as consequent of a single conditional statement" ([5], p. 202).

This is a mistake. To be sure, any modern quantificational calculus will employ meta-linguistic statements such as the schema of universal instantiation, and here we 
find restrictions. One puts

( $\mu$ ) $A \mu \supset A[t \mid \mu]$, where $t$ is free for $\mu$ in the wff $A$.

In the schema, ' $A$ ' is a meta-linguistic letter restricted to well-formed formulas of the object language and ' $t$ ' a schematic letter restricted to terms of that language. But these are not at all the sort of restrictions that Russell finds objectionable. Put succinctly, the doctrine of the unrestricted variable simply demands that the objectlanguage of any calculus for logic should embrace one style of variables-viz., entity or individual variables. ${ }^{6}$ Russell's point is that even if there are distinct sorts of entities - universals, particulars, propositions - a calculus for logic should not adopt many sorted variables such as propositional variables, predicate variables, or particular variables. If $p$ is supposed to be a special propositional variable, then Russell argues, " $p$ implies, for all $p$ and $q, q$ 's implying $p$ " really just means " $x$ 's and $y$ 's being propositions implies, for all $x$ and $y, x$ 's implying $y$ 's implying $x$." Again, if ' $R$ ' is supposed to be a special relation variable, then Russell argues, "For all $R, R a b$ " just means " $x$ 's being a relation implies, for all $x, a$ 's and $b$ 's belonging to $x$ " (10], p. 87).

There are, to be sure, certain difficulties in Russell's approach. As he realizes, he will need to enlist such primitives as " $x$ is a concept (property or relation)" (10, p. 86). Moreover he will need an infinite stock of $n$-place relations so that, for example, for any proposition ' $R(a, b)$ ' there is another proposition ' $R *(a, b, R)$ ' which is logically equivalent to it. Russell acknowledges this in his discussion of the Bradley regress, finding the infinity undeniable but wholly innocuous so long as the predicational nature of concepts is accepted (10], p. 100). Accordingly, Russell implicitly accepts

$$
x_{1}, \ldots, x_{n} \text { exemplify } y
$$

as a primitive logical notion. But in any case, nothing in Russell's adoption of one style of variable shows that he did not (and could not) view his system as a calculus in a modern sense.

The above arguments dismissed, what of Russell's own rejection of metalinguistic proofs? In Principles, he rejects Hilbert-style independence proofs. ${ }^{7}$ Why? Does this not show that the very concept of going outside to the meta-language was absurd to Russell? No. Russell, as Frege before him, rejected Hilbert-style independence proofs on grounds that since such proofs must rob the primitive symbols of their intended interpretations, the proofs cannot be germane when those primitives have their intended interpretations. Independence is a syntactic matter concerning derivability, and derivability within a calculus for logic must not be confused with implication. It is important that the former track the latter. After all, one can adopt rules for a formal calculus that do not track logic at all. Nonetheless, results concerning derivability can be useful, and Frege and Russell were simply overzealous in their concern that changing the meaning of the logical particles of a purported formal calculus for logic severs the connection between the calculus and logic itself.

Russell also, and infamously, rejected the use of inductive proofs to get results about his calculus. With rules and schemata of his sentential calculus confined to quantifier-free well-formed formulas, Russell wanted to show in *9 of the 1910 Principia Mathematica, that by adding a few inference rules and axiom schemata the resulting system was as strong as $* 10$ (a system which allowed the sentential schemata 
to apply to all well-formed formulas). He explicitly rules out using mathematical induction ([18], p. 130). This is easily explained, however. One cannot, without begging questions against logicism, suppose that the notions required for the articulation of a calculus for logic are distinctly mathematical notions. All the same, it would be equally question-begging in favor of logicism to employ what have been traditionally thought to be distinctly mathematical notions, like mathematical induction, in setting out a calculus for logic. Logicism must demonstrate that one can deduce mathematical induction from within a system whose basis is grounded upon intuitions of pure logic alone.

The fact is that Russell was explicitly concerned with questions of completeness and independence ([10, p. 15). Though infamously sloppy about use and mention, we shall see that he did have a sense of what we call the difference between metalanguage and object- language, and endeavored to set forth axioms and inference rules of a formal calculus (in the modern sense) for logic.

2 Russell's propositional calculus The phrase propositional calculus is commonly used to refer to a sentential calculus. Russell's use is nonstandard, for it refers to a system which is analogous to a higher-order calculi-that is, a system which permits bindable predicate variables in subject as well as predicate positions. At first blush, this seems surprising, for we have seen that Russell embraces only individual variables. But extended by the logical constant, say, ' $C$ ' for the property of being concept (property or relation), and the constants $\left\ulcorner P^{n}\right\urcorner, n \in N$, for the $n$-adic relations of exemplification, the presence of only individual variables poses no untoward limitations. To transcribe "Every human is mortal," Russell can put

$$
p^{2}(x, \text { Humanity }) \supset_{x} P^{2}(x, \text { Mortality }),
$$

which, read literally, says " $x$ 's exemplifying Humanity implies, for all $x, x$ 's exemplifying Mortality." Similarly, Russell can transcribe "Every entity has every property" with,

$$
C(x) . \supset_{x, y} \cdot P^{2}(y, x)
$$

which reads, " $x$ 's being a concept implies, for all $x$ and $y, y$ 's exemplifying $x$." So we can see that the system has an expressive capacity beyond that of a sentential logic.

Indeed, Russell intended yet more. He wanted a theory of classes as well. For this purpose he took Peano's ' $\in$ ' (for membership) and ' $\ni$ ' (for 'such that') as primitive signs so that $\ulcorner x \in z \ni A z\urcorner$ is a well-formed formula which reads " $\mathrm{x}$ is a member of the class of all $z$ such that $\ldots, z, \ldots$. " He adopts some primitive principles for classes ([10], p. 20) as well, and in virtue of the theory of classes, he hopes to define the identity sign, and thus writes:

Identity which occurs here may be defined as follows: $x$ equals $y$ if $y$ belongs to every class to which $x$ belongs, in other words $x$ is a $\mu$ implies $y$ is a $\mu$ for all values of $\mu$. (ibid.)

Of course, this requires a comprehension principle for classes (or concepts). Russell had none to offer. He had discovered the paradoxes surrounding the unbridled assumption that every open well-formed formula comprehends an attribute and a class. 
Russell intended the theory of extension as a part of his calculus for logic. But in Principles his ideas on the paradoxes were in a state of flux.

In what follows, our purpose is to show completeness with respect to analogs of sentential tautologies. For this, we need only a fragment of Russell's system. All the same, it is worth remembering the richness of the sort of system that Russell calls his Calculus of Propositions.

Russell's system originates from a fateful encounter with Peano at a congress in Paris in 1900. It was, as Russell later recalled in My Mental Development, "the most important year of my intellectual life." Russell was struck by the techniques and logical apparatus demonstrated at the congress. He found the precision afforded by Peano's notation of inestimable value, and was very much influenced by the philosophy of mathematics espoused by Peano and his school. Peano introduced the expression, ' $p \supset q$ ', writing,

On pourrait indiquer la relation $p \supset q$ par la signe $q C p$ qu' on lira " $q$ est conséquence $p . "$ (77, p. 26)

Peano reads the symbol as " $q$ is a consequence of $p$," and $p$ is called the "hypothesis." Unfortunately, he was in the habit of using context to determine the meaning of some of his signs. For instance, he used '=' to express a number of distinct relations; sometimes it expressed identity, other times equivalence, and still other times it was used in stipulative definitions. In the case of ' $p \supset q$ ', he read it as "de la $p$ deduit la $q$," a reading which he viewed as warranting detachment when the "hypothesis" is true.

Peano introduced an expression for universal quantification (formal implication) as well. He had some innovations similar to Frege's begriffsschrift, distinguishing free and bound variables and introducing a notation of quantification which made the expression of scope possible:

Soient $p$ et $q$ des propositions contenant des lettres variables $x \ldots z$. Noûs ècrirons

$$
p . \supset_{x \ldots z} \cdot q
$$

pour indiquer la proposition "de $p$ on déduit, quels que soint $x \ldots z$, la $q$." (Peano [7], p. iii)

The quantifier is here subscripted to the conditional sign and thus the name 'formal implication' - as if we have some new form of implication.

Following Peano, Principles adopts the sign ' $\supset$ ' as a primitive dyadic predicate constant which stands for the relation of material implication. Similarly, ' $\supset_{\mu_{1}, \ldots, \mu_{n}}$ ' is adopted for formal implication. ${ }^{8}$ Let us use ' $\alpha$ ', ' $\beta$ ', and ' $\delta$ ' as meta-linguistic letters for terms, and ' $A$ ', ' $B$ ', and ' $C$ ' as meta-linguistic letters for well-formed formulas. The well-formed formula $\ulcorner\alpha \supset \beta\urcorner$, is read $\alpha$ implies $\beta$, and the positions of $\alpha$ and $\beta$ here are subject positions. Thus, ' $\supset$ ' stands for a relation. The relata can be any two entities, propositions or otherwise. Propositions are mind independent entities in Russell's view, and some are true and others false. Thus there is a proposition ' $x \supset y$ ' no matter what entities $x$ and $y$ are; it may not, however, be a true proposition.

To make the syntax of Russell's dyadic predicate ' $\supset$ ' salient, it is useful to introduce nominalizing braces ' $\{$ ' and ' $\}$ '. By using the braces, a well-formed formula 
$A$ is made into a term $\ulcorner\{A\}\urcorner$. Thus,

$$
x \supset\{y \supset z\}
$$

would be a formula. Russell did not employ such braces in Principles. Instead, he took subject position to be sufficient to mark the nominalizing transformation. Russell's approach is convenient. Braces can be dropped for subject position, and dots and brackets can be used for punctuation. Thus, one can write

$$
x . \supset . y z \text {. }
$$

We shall follow this. But readers should be wary not to confuse ' $\supset$ ' with the modern conditional sign ' $\rightarrow$ ' which is flanked by well-formed formulas not terms. Unfortunately, the confusion is easy to fall into. Russell often uses the letters ' $p$ ', ' $q$ ', ' $r$ ', ' $s$ ', ' $t$ ', and these appear as if they are special letters for propositions. They are not.' They are just variants of ' $x$ ', ' $y$ ', ' $z$ ', and so forth, which are (now commonly used as) individual variables. In Principles, Russell uses the words 'term', 'unit', 'one', 'entity', and 'logical subject' synonymously with the word 'individual'. The fundamental doctrine of the work is that whatever is, (be it a proposition or otherwise) is an individual. Logic treats all entities alike as values of its wholly unrestricted variables. Thus, there are no special primitive propositional variables in the work. To remind the reader, I shall replace Russell's letters with individual variables ' $x$ ', ' $y$ ', ' $z$ ', and so on.

Now Russell decides that only propositions stand in true inferential relationships. No proposition ' $x \supset y^{\prime}$ can be true unless both $x$ and $y$ are propositions. Accordingly, Russell notes that he need not introduce a primitive constant ' $x$ is a proposition'. Rather he need only put

$$
\left.x \text { is a proposition }=_{d f} x \supset x .(10], \mathrm{p} .15\right)
$$

Russell never sets out his calculus for logic formally and aspects of it are scattered throughout Principles. The first ten fundamental principles are as follows:

$$
\begin{aligned}
& \mathrm{Pp}_{1} \quad x \supset y . \supset_{x, y} . \quad x \supset y \\
& \mathrm{Pp}_{2} \quad x \supset y . \supset_{x, y} \quad x \supset x \\
& \mathrm{Pp}_{3} \quad x \supset y . \supset_{x, y} . \quad y \supset y \\
& \mathrm{Pp}_{4} \quad \text { A true hypothesis in an implication may be } \\
& \text { dropped, and the consequent asserted. } \\
& \text { Simplification } \mathrm{Pp}_{5} \quad x \supset x: \supset_{x, y}: \quad y \supset y . \supset .(x y . \supset . x) \\
& \text { Composition } \mathrm{Pp}_{7} \quad x \supset y: \supset_{x, y, z}: x \supset z . \supset .(x . \supset . y z) \\
& \text { Importation } \mathrm{Pp}_{8} \quad y \supset y: \supset_{x, y, z}: z \supset z . \supset .(x . \supset . y \supset: \supset: x y . \supset . z) \\
& \text { Exportation } \quad \mathrm{Pp}_{9} \quad x \supset x: \supset_{x, y, z}: y \supset y . \supset .(x y . \supset . z: \supset: x . \supset . y \supset z) \\
& \text { Reduction } \mathrm{Pp}_{10} \quad x \supset x: \supset_{x, y}: y \supset y . \supset .(x \supset y . \supset . x: \supset: x)(\text { [10], p. 6) }
\end{aligned}
$$$$
\text { Syllogism } \mathrm{Pp}_{6} \quad x \supset y: \supset_{x, y, z}: y \supset z . \supset . x \supset z
$$

(The expression ' $x y$ ' is used for conjunction.)

Special attention must be paid to Russell's $\mathrm{Pp}_{4}$. Unlike the other principles, it is an inference rule similar to the modern Modus Ponens. Strictly speaking Peano had no formal calculus for logic, since he had no explicit inference rules. Frege informed 
him of this failing on several occasions to no avail. Reading ' $\supset$ ' as "implies" in one context and "therefore" in another, Peano likely thought that detachment need not be explicitly stated as a rule. Russell, on the contrary, made a significant attempt at providing the missing rules.

Russell was aware of the special status of $\mathrm{Pp}_{4}$ among his "principles." Concerning the principle, he wrote,

This is a principle incapable of formal symbolic statement, and illustrating the essential limitations of formalism - a point to which I shall return at a later stage. (10], p. 16)

The "essential limitation of formalism," as Russell put it, is precisely Russell's way of saying that an inference rule is meta- linguistic and not itself an axiom among others. Russell speaks at length to the point in his discussion of Carroll's paradox "What the Tortoise said to Achilles" (10], p. 35). He says that we need the notion of therefore which is quite distinct from the notion of implies. The notion of therefore renders an inference license warranting detachment; the notion of implies does not.

The principle $\mathrm{Pp}_{4}$ is important in another way as well. In stating his axioms, Russell does not index his sign ' $\supset$ ' as we have above. For example, he states $\mathrm{Pp}_{1}$ as follows:

If $p$ implies $q$, then $p$ implies $q$; in other words, whatever $p$ and $q$ may be, ' $p$ implies $q$ ' is a proposition. (10], p. 16)

But Russell is just following the mathematical convenience (commonplace as well in Peano's notation) of omitting indices. In a footnote he makes this clear.

Note that the implications denoted by if and then, in these axioms, are formal while those denoted by implies are material. (ibid.)

So Russell's axioms are formal implications. Accordingly, he needs a quantification theory for his calculus to work. For this reason, Russell allows $\mathrm{Pp}_{4}$ to have an application to general formulas:

Another form in which the principle is constantly employed is the substitution of a constant, satisfying a hypothesis, in the consequent of a formal implication. If $\varphi x$ implies $\theta x$, for all values of $x$, and if $a$ is a constant satisfying $\varphi x$, we can assert $\theta a$, dropping the true hypothesis $\varphi a$. (10], p. 35)

To accommodate both forms, $\mathrm{Pp}_{4}$ can be written as follows. From

$$
\alpha \supset \mu_{0}, \ldots, \mu_{n} \beta \text { and } \alpha\left[t_{1}\left|\mu_{1}, \ldots, t_{n}\right| \mu_{n}\right]
$$

infer

$$
\beta\left[t_{1}\left|\mu_{1}, \ldots, t_{n}\right| \mu_{n}\right]
$$

where

$$
t_{i} \text { is free for } \mu_{i} \text { in } \alpha \text { and } \beta, 0 \leq i \leq n .
$$

We let $\alpha[t \mid \mu]$ be $t$ when the term $\alpha$ is the variable $\mu$. The expression $\left\ulcorner\alpha \supset \mu_{0}, \ldots, \mu_{n} \beta\right\urcorner$ is for any implication, formal (when $n \neq 0$ ) or material (where $n=0$ ).

Now in addition to the two forms of $\mathrm{Pp}_{4}$, Russell has the rule of universal generalization. It is stated informally: 
So long as any term in our proposition can be turned into a variable, our proposition can be generalized; and so long as this is possible, it is the business of mathematics to do it. (10], p. 7)

The precise formulation of this rule is important. It would not do to characterize it as:

If $\alpha \supset \beta$ is a thesis, so is $\alpha \supset_{\mu} \beta$,

where $\mu$ is an entity variable free in $\alpha$ or $\beta$ (or equal to $\alpha$ or $\beta$ ). Such a formulation, together with $\mathrm{Pp}_{4}$, would not suffice to generate quantification theory. Moreover, such a form would make universal generalization under the hypothesis of a conditional proof illegitimate. Russell's statement of the "other form" of $\mathrm{Pp}_{4}$ suggests that he allows conditional proofs. Verification of this comes if we look at Peano.

Consider Peano's 1889 The Principles of Arithmetic. As usual, Peano omits indices on his sign ' $\supset$ ', writing,

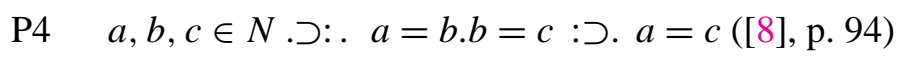

instead of

P4 $\quad a, b, c \in N . \supset_{a, b, c}: . a=b \cdot b=c: \supset . a=c$.

But examine the following "proof."

Theorem $13 \quad a, b, c \in N . a=b . b=c . c=d: \supset: a=d$

Hyp.P4 : : $a, c, d \in N . a=c . c=d . P 4: \supset:$ Thes

The idea of the proof seems to be to assume the antecedent of the theorem to be shown. On the basis of the assumption, Peano's axiom P4 can be applied by means of Russell's rule $\mathrm{Pp}_{4}$. This yields,

$$
a, c, d \in N \& a=c \& c=d .
$$

Calling again upon $\mathrm{P} 4$ and using Russell's $\mathrm{Pp}_{4}$, one arrives at $a=d$. By an implicit rule of conditional proof then, Peano has

$$
\text { Hyp } \supset a=d \text {. }
$$

The final result is obtained by universal generalization. If this is correct, Peano (albeit tacitly) employs an inference rule legitimating deduction from a hypothesis. Since Peano's calculus was Russell's paradigm and it is clear that Russell intended Principles to amend and correct Peano's proof techniques, it seems likely that he allowed derivations under a hypothesis. When it came to the question of quantification rules, Peano did express the following concern:

The indices to the sign $\supset$ satisfy laws which have not yet been sufficiently studied. This theory, already abstruse in itself, becomes even more so unless the rules are accompanied by examples...([7, $\S 18)$

In a letter to Peano, Frege noted that he had already given the proper rules in his 1879 Begriffschrift and, being that they are few in number and clear, knew of no reason they should be said to be abstruse (Frege [4], p. 11). But in allowing conditional proof, Peano and Russell faced issues that Frege did not; they must formulate the rule of universal generalization in a way that demarcates when generalization under the scope 
of a hypothesis is legitimate. This may well explain why Peano found the articulation of rules for quantification "abstruse." Unfortunately, formulations of Universal Generalization and Conditional Proof are hard to exact from Russell's writings of the period. In "The theory of implication" of 1905/6, Russell states,

*.7.11 What is true of any is true of all.

Alternatively, he puts,

If $\varphi y$ is true however $y$ is chosen, then $(x) . \varphi x$ is true. (11], p. 195)

This, of course, is misleading. ${ }^{10}$ Consider

$\begin{array}{llr}\text { 1. } & (x)(A x \supset B x) & \text { Hyp } \\ \text { 2. } & (x) A x & \text { Hyp } \\ \text { 3. } & A x \supset B x & 1, \mathrm{UI} \\ \text { 4. } & A x & 2, \mathrm{UI} \\ \text { 5. } & B x & 4,3, \mathrm{MP} \\ \text { 6. } & (x) B x & 5, \mathrm{UG} \\ \text { 7. } & 2 \supset 6 & \\ \text { 8. } & 1 \supset 7 & \end{array}$

Here line 5 may not be true at all. Russell seems to be aware of this. Perhaps, then Russell's point was that a well-formed formula of the form $\varphi$ (e.g., our line 5) can be said to be "true" in the sense that it would be arrived at in a similar demonstration no matter what variable is chosen instead of y. In what follows, I shall adopt the following on Russell's behalf.

$$
\text { (Universal Generalization) From } \alpha \supset_{v_{o}, \ldots, v_{n}} \beta \text { infer } \alpha \supset_{\mu, v_{o}, \ldots, v_{n}} \beta \text {, }
$$

where $\mu$ is an individual variable which is free in $\alpha \supset v_{o}, \ldots, v_{n} \beta$, and which does not occur free in any hypotheses within whose scope $\alpha \supset_{\mu, v_{o}, \ldots, v_{n}} \beta$ occurs.

3 Russell's definitions Russell put forth definitions of tilde, conjunction, and disjunction signs in Principles. There is a good discussion of the definitions in Byrd [1]. The following diverges somewhat from Byrd's renditions, however. What Byrd takes as errors I take as slips or infelicities of expression. Russell begins with the conjunction sign (i.e., juxtaposition) as follows:

If $p$ implies $p$, then if $q$ implies $q$, then $p q$ (the logical product of $p$ and $q$ ) means that if $p$ implies that $q$ implies $r$, then $r$ is true. (10], p. 17)

Unfortunately, there is a slip. Russell read " $r$ " as if it stood only for propositions. Russell corrects this in an adjoining sentence of clarification, writing:

In other words, if $p$ and $q$ are propositions, then their joint assertion is equivalent to saying that every proposition is true which is such that the first implies that the second implies it. (ibid.)

Accordingly Russell has:

$x \supset x: \supset_{x y}: y \supset y . \supset$. [ $\left[x y\right.$ is equivalent to $\left.z \supset z . \supset_{z} \cdot(x . \supset . y \supset z: \supset: z)\right]$

We are still faced with difficulties. First, the definition is conditional; and second, what is the import of "means that" (or "is equivalent to" as Russell uses it above)? 
Likely, the answer is simply that Russell felt his readers would find a conjunction sign between entity variables to be absurd. How, for example, can one assert 'Frege and Russell'? And obscure as it seems, would one be able to infer Frege from the conjunction? Russell's Simplification avoids this problem by restriction to propositions. There is no derived inference rule: From $x y$, infer $x$. A variable cannot occur isolated on a line of a demonstration. All the same, Russell may have felt he needed to assure readers that his conjunction does parallel the normal truth-conditions of 'and'. In his effort to assure readers, Russell adopted the conditional definition.

If this is right then we can be content to drop Russell's conditional definitions and his locution "is equivalent to". It would not suffice, however, to adopt the definition schema

$$
A B={ }_{d f} \mu \supset \mu . \supset_{\mu} .(A . \supset . B \supset \mu: \supset: \mu),
$$

where $A$ and $B$ are any well-formed formulas of the language. This defines conjunction where nominalized well-formed formulas (for material or formal implications) are concerned. Russell's axioms (e.g., Simplification) would not comport with definition, for they allow the expression of the conjunction sign (juxtaposition) with individual variables (Byrd [1], p. 351). Our solution is to adopt the definition schema

$$
\alpha \beta={ }_{d f} \mu \supset \mu . \supset_{\mu} \cdot(\alpha . \supset . \beta \supset \mu: \supset: \mu),
$$

for any terms $\alpha, \beta$.

Russell next defines disjunction. He introduces no special sign, but we shall adopt ' $\vee$ ' on his behalf. He writes,

' $p$ or $q$ ' is equivalent to “ " $p$ implies $q$ ” implies $q$ '. (10], p. 17)

At first blush, Russell seems to have offered a definition which is not conditional. His statement of clarification, however, suggests that he again slipped by reading ' $p$ ' and ' $q$ ' as if they stood for nominalized well-formed formulas (for propositions). He writes,

It is easy to persuade ourselves of this equivalence since a false proposition implies every other. (ibid.)

We shall avoid the conditional definition putting

$$
\alpha \vee \beta={ }_{d f} \alpha \supset \beta . \supset . \beta
$$

The same point applies to the definition of the negation sign. Russell introduces no sign, but writes

...we proceed to the definition of negation; not- $p$ is equivalent to the assertion that $p$ implies all propositions; i.e., that ' $r$ implies $r$ ' implies ' $p$ implies $r$ ' whatever $r$ may be. ([10] p. 18)

Appearances to the contrary, Russell once again intends a conditional definition. We see this because the definition is accompanied by a footnote which reads,

The principle that false propositions imply all propositions solves Lewis Carroll's logical paradox in Mind, N.S. No. 11 (1894). (ibid.) 
So again there is a slip, here with regard to the use of 'p'. Russell wants:

$$
x \supset x . \supset_{x} \text {. [ not- } x \text { is equivalent to } z \supset z . \supset_{z} . x \supset z \text { ] }
$$

Evidently, Russell wanted his readers to be sure to understand that his definition will yield the usual,

$$
x \text { implies not not } x
$$

where $x$ is a proposition, even though it is not a thesis otherwise. All the same, we are dropping the conditional definitions, and so (adopting ' $\neg$ ' on Russell's behalf) we have

$$
\neg \alpha=_{d f} \mu \supset \mu . \supset_{\mu} . \alpha \supset \mu
$$

as our definition schema. No biconditional sign is introduced or defined, and we must be wary not to confuse this matter with the locution 'is equivalent to' in Russell's statements of definition. Curiously, in discussing the equivalence of relations, Russell does suggest an awareness of Peano's approach - that is, of introducing a biconditional sign with the definiens the conjunction of two conditionals ([10], p. 24). Peano used ' $=$ ' to express a number of distinct relations, while Pieri used ' $\equiv$ ' for definitions, reserving '=' for identity. Perhaps, Russell had not settled on a sign.

The existential quantifier remains. In a letter to Frege of May 24, 1903 we find Russell adopting the notation of $\ulcorner(\mu) A \mu\urcorner$ and defining Peano's notation which indexes the conditional sign. This provides a means of avoiding having to put $\ulcorner-A x \supset x$ $A x\urcorner$ when $\ulcorner(x) A x\urcorner$ is wanted. Russell also puts

$$
(\exists x) \cdot \varphi x=_{d f}-\{(x) .-\varphi x\} . \text { (McGuinness [6], p. 159) }
$$

There are some hints of this in Principles. Peano had put

$$
\exists a=_{d f}-(a=\bigwedge) .
$$

His symbol ' $\exists$ ' is not a variable binding operator. It flanks a class term ' $a$ ' and is used to say that the class $a$ is not empty. Now in Principles, Russell explains that a class $a$ exists when it (i.e., when the class-concept of which $a$ is the would-be extension) is nonempty (i.e., is exemplified). Haplessly, Russell uses 'an $a$ ' for the class-concept and, running together the class concept expression with the class term (an error he chides Peano with), finds himself writing:

A class is said to exist when it has at least one term. $A$ formal definition of this is: $a$ is an existent class when and only when any proposition is true provided " $x$ is an $a$ " always implies it whatever value we may give to $x$. ([10], p. 21)

Where ' $\hat{x}(x$ is an $a)$ ' is a class term, what Russell intends is

$$
\exists \hat{x}(x \text { is an } a)=_{d f}-(x)-(x \text { is an } a) .
$$

It was not long before this would become

$$
\exists ! \alpha=_{d f}(\exists x)(x \in \alpha)
$$

which was put in Principia as *24.03 [17]. So the passage ${ }^{11}$ from Principles certainly foreshadows Russell's later definition of the existential quantifier. 
No rule for definitions is adopted explicitly. But Russell was well aware that since definitions are but notational conveniences, definiens and definiendum may replace one another in any context. He could easily read of this from most any volume of Peano's Formulaire.

4 Completeness of the propositional calculus with respect to propositional analogs of sentential tautologies Let us characterize the terms and well-formed formulas of the fragment of the system of Principles we shall call ' $(P)$ '. The variables are to be ' $x$ ', ' $y$ ', ' $z$ ', ' $u$ ', ' $v$ ' , ' $w$ ', and all primes so that there is an infinite stock. Primitive signs are: brackets, '(' and ')'; braces, ' $\{$ ' and ' $\}$ '; the sign ' $\supset$ '; and the formal implication sign ' $\supset \mu_{1}, \ldots, \mu_{n}$ ' where each $\mu_{i}$ is an individual variable, $1 \leq i \leq n$. The terms and well-formed formulas are characterized together. The well-formed formulas are thus: (1) where $\alpha, \beta$ are terms, and $\mu_{i}, 1 \leq i \leq n$ are individual variables, $\ulcorner\alpha \supset \beta\urcorner$ and $\left\ulcorner\alpha . \supset \mu_{1}, \ldots, \mu_{n} . \beta\right\urcorner$ are well-formed formulas; and (2) there are no other well-formed formulas. The terms are: (1) all individual variables are terms; (2) where $A$ is a wellformed formula, $\ulcorner\{A\}\urcorner$ is a term; and (3) there are no other terms.

The axioms and transformation rules are reiterated below for convenience:

$$
\begin{aligned}
& \mathrm{Pp}_{1} \quad x \supset y . \supset_{x, y} . \quad x \supset y \\
& \mathrm{Pp}_{2} \quad x \supset y . \supset_{x, y} . \quad x \supset x \\
& \mathrm{Pp}_{3} \quad x \supset y . \supset_{x, y} . \quad y \supset y \\
& \mathrm{Pp}_{4} \quad \text { From } \alpha \supset \mu_{0}, \ldots, \mu_{n} \beta \text { and } \alpha\left[t_{0}\left|\mu_{0}, \ldots, t_{n}\right| \mu_{n}\right] \text {, } \\
& \text { infer } \beta\left[t_{0}\left|\mu_{0}, \ldots, t_{n}\right| \mu_{n}\right] \text {, where } t_{i} \text { is free for } \\
& \mu_{i} \text { in } \alpha \text { and } \beta, 0 \leq i \leq n \text {. } \\
& \text { Simplification } \mathrm{Pp}_{5} \quad x \supset x: \supset_{x, y}: y \supset y . \supset .(x y . \supset . x) \\
& \text { Syllogism } \quad \mathrm{Pp}_{6} \quad x \supset y: \supset_{x, y, z}: y \supset z . \supset . \quad x \supset z \\
& \text { Composition } \mathrm{Pp}_{7} \quad x \supset y: \supset_{x, y, z}: x \supset z . \supset .(x . \supset . y z) \\
& \text { Importation } \mathrm{Pp}_{8} \quad y \supset y: \supset_{x, y, z}: z \supset z . \supset \text {. ( }(x . \supset . y \supset: \supset: x y . \supset . z) \\
& \text { Exportation } \quad \mathrm{Pp}_{9} \quad x \supset x: \supset_{x, y, z}: y \supset y . \supset .(x y . \supset . z: \supset: x . \supset . y \supset) \\
& \text { Reduction } \mathrm{Pp}_{10} \quad x \supset x: \supset_{x, y}: y \supset y . \supset .(x \supset y . \supset . x: \supset: x)
\end{aligned}
$$

(UG) Rule of Universal Generalization

From $\alpha \supset_{v_{0}, \ldots, v_{n}} \beta$, infer $\alpha \supset_{\mu, v_{0}, \ldots, v_{n}} \beta$, where $\mu$ is an individual variable which is free in $\alpha \supset_{v_{0}, \ldots, v_{n}} \beta$, and which does not occur free in any hypotheses within whose scope $\alpha \supset_{\mu, v_{0}, \ldots, v_{n}} \beta$ occurs.

(CP) Rule of Conditional Proof

$$
\begin{aligned}
& \text { i. } A \quad \text { Hyp } \\
& \text { m. } B \\
& m+1 . \quad A \supset B \quad i-m, \mathrm{CP} \\
& \neg \alpha=_{d f} \quad \mu \supset \mu . \supset_{\mu} \cdot \alpha \supset \mu \\
& \alpha \beta={ }_{d f} \quad \mu \supset \mu .: \supset_{\mu}: \alpha \supset \beta . \supset . \mu: \supset: \mu \\
& \alpha \vee \beta={ }_{d f} \quad \alpha \supset \beta . \supset . \beta
\end{aligned}
$$

For reasons that will become clear later on, we add 


$$
\begin{aligned}
f & { }_{d f} & \mu \supset \mu . \supset_{\mu} \mu \\
\alpha \& \beta & =_{d f} & \alpha . \supset . \beta \supset f: \supset: f \\
-\alpha & { }_{d f} & \alpha \supset f
\end{aligned}
$$

Call this system '( $\mathrm{P})$ '.

The presence of the rule of conditional proof assures the adequacy of Russell's quantification theory. From $\mathrm{Pp}_{1}$ and inference rule (CP) we can arrive at the following theorem for universal instantiation.

Theorem Schema $(a)$

$\vdash \alpha \supset \mu_{1}, \ldots, \mu_{n} \beta$.つ. $\alpha\left[t_{1}\left|\mu_{1}, \ldots, t_{n}\right| \mu_{n}\right] \supset \beta\left[t_{1}\left|\mu_{1}, \ldots, t_{n}\right| \mu_{n}\right]$,

where $t_{i}$ is free for $\mu_{i}$ in $\alpha$ and $\beta, 1 \leq i \leq n$.
1. $\alpha \supset_{\mu_{1}, \ldots, \mu_{n}} \beta$
2. $\quad \mid \alpha\left[t_{1}\left|\mu_{1}, \ldots, t_{n}\right| \mu_{n}\right]$
3. $\quad \beta\left[t_{1}\left|\mu_{1}, \ldots, t_{n}\right| \mu_{n}\right]$
Hyp
Нyp
4. $\alpha\left[t_{1}\left|\mu_{1}, \ldots, t_{n}\right| \mu_{n}\right] \supset \beta\left[t_{1}\left|\mu_{1}, \ldots, t_{n}\right| \mu_{n}\right] \quad 2,3, \mathrm{CP}$
5. $\alpha \quad \supset \mu_{1}, \ldots, \mu_{n} \beta$.つ. $\alpha\left[t_{1}\left|\mu_{1}, \ldots, t_{n}\right| \mu_{n}\right] \supset$$$
\beta\left[t_{1}\left|\mu_{1}, \ldots, t_{n}\right| \mu_{n}\right] \quad 1-4, \mathrm{CP}
$$

This validates a derived rule, DR(UI), for Universal Instantiation. We also have a version of Theorem Schema $_{(a)}$ for single instantiations.

Theorem Schema $\left(a^{\circ}\right)$

$\vdash \alpha \supset_{\mu_{1}, \mu_{2}, \ldots, \mu_{n}} \beta$.つ. $\alpha\left[t \mid \mu_{1}\right] \supset_{\mu_{2}, \ldots, \mu_{n}} \beta\left[t \mid \mu_{1}\right]$,

where $t$ is free for $\mu_{1}$ in $\alpha$ and $\beta$.
1. $\alpha \supset \mu_{1}, \mu_{2}, \ldots, \mu_{n} \beta$
Hyp
2 .$$
\alpha\left[t\left|\mu_{1}, \mu_{2}\right| \mu_{2}, \ldots, \mu_{n} \mid \mu_{n}\right] \supset
$$$$
\beta\left[t\left|\mu_{1}, \mu_{2}\right| \mu_{2}, \ldots, \mu_{n} \mid \mu_{n}\right]
$$
3. $\alpha\left[t \mid \mu_{1}\right] \supset \beta\left[t \mid \mu_{1}\right]$
2
4. $\quad \alpha\left[t \mid \mu_{1}\right] \supset_{\mu_{2}, \ldots, \mu_{n}} \beta\left[t \mid \mu_{1}\right]$
3, UG
5. $\alpha \quad \supset_{\mu_{1}, \ldots, \mu_{n}} \beta$.Ј. $\alpha\left[t \mid \mu_{1}\right] \supset_{\mu_{2}, \ldots, \mu_{n}} \beta\left[t \mid \mu_{1}\right]$

We shall call both by the name "DR(UI)" in what follows. Next we have a schema for the quantifier.

Theorem Schema $(b)$

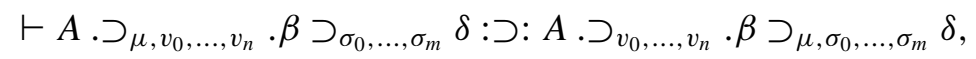
where $\mu$ is not free in $A$.

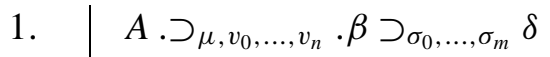
2. $\quad A . \supset . \beta \supset \sigma_{0}, \ldots, \sigma_{m} \delta$
3. $\mid A$
4. $\quad \beta \supset \sigma_{0}, \ldots, \sigma_{m} \delta$
5. $\quad \beta \supset \mu, \sigma_{0}, \ldots, \sigma_{m} \delta$
6. $\quad A . \supset . \beta \supset \mu, \sigma_{0}, \ldots, \sigma_{m} \delta$
7. $\quad A . \supset v_{0}, \ldots, v_{n} . \beta \supset \supset_{0}, \ldots, \sigma_{m} \delta$
8. $\quad A \quad \supset_{\mu, v_{0}, \ldots, v_{n}} . \beta \supset_{\sigma_{0}, \ldots, \sigma_{m}} \delta: \supset: A . \supset_{v_{0}, \ldots, v_{n}}$. $\beta \supset_{\mu, \sigma_{0}, \ldots, \sigma_{m}} \delta$
$2-6, \mathrm{CP}$
$6, \mathrm{UG}$
$1-7, \mathrm{CP}$ 
This validates a derived rule based on the schema. We now have all that is needed for quantification theory. From $\mathrm{Pp}_{1}$ we know that every material implication implies itself. But we can now also prove that formal implication implies itself.

Theorem Schema $(c)$

$$
\begin{aligned}
& \vdash \alpha \supset \mu_{1}, \mu_{2}, \ldots, \mu_{n} \beta . \supset . \alpha \supset \mu_{1}, \mu_{2}, \ldots, \mu_{n} \beta \\
& \text { 1. } \alpha \supset_{\mu_{1}, \mu_{2}, \ldots, \mu_{n}} \beta . \supset . \alpha \supset_{\mu_{2}, \ldots, \mu_{n}} \beta \quad \quad \text { Theo }_{\left(\mathrm{a}^{\circ}\right)}
\end{aligned}
$$

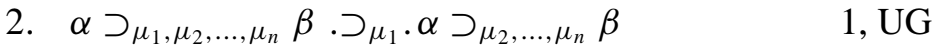

$$
\begin{aligned}
& \text { 3. } \alpha \supset_{\mu_{1}, \mu_{2}, \ldots, \mu_{n}} \beta \text {. } \supset . \alpha \supset_{\mu_{1}, \mu_{2}, \ldots, \mu_{n}} \beta \quad 2, \text { Theo }_{(\mathrm{b})}, \mathrm{Pp}_{4}
\end{aligned}
$$

Now from Simplification $\left(\mathrm{Pp}_{5}\right)$, and $\mathrm{DR}(\mathrm{UI})$ one can arrive at

$$
\begin{aligned}
& \vdash(x \supset y)(z \supset w) . \supset_{x, y, z, w \cdot x \supset y}
\end{aligned}
$$

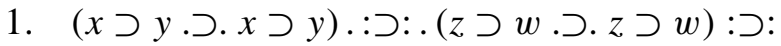

$$
\begin{aligned}
& (x \supset y)(z \supset w) . \supset . x \supset y \\
& \text { simp, DR(UI) } \\
& \text { 2. } x \supset y . \supset . x \supset y \\
& \mathrm{Pp}_{1}, \mathrm{DR}(\mathrm{UI}) \\
& \text { 3. }(z \supset w . \supset . z \supset w): \supset:(x \supset y)(z \supset w) \text {. }(x . x \supset y \\
& 1,2, \mathrm{Pp}_{4} \\
& \text { 4. } z \supset w . \supset . z \supset w \\
& \text { 5. }(x \supset y)(z \supset w) \text {. } \supset . x \supset y \\
& \mathrm{Pp}_{1}, \mathrm{DR}(\mathrm{UI}) \\
& 3,4, \mathrm{Pp}_{4} \\
& \text { 6. }(x \supset y)(z \supset w) . \supset_{x, y, z, w} \cdot x \supset y \\
& 5, \mathrm{UG}
\end{aligned}
$$

So any two well-formed formulas, each the form of a material implication, will be such that their conjunction obeys Simplification. This also holds for any two formal implications.

$$
\begin{aligned}
& \vdash\left(\alpha \supset_{\mu_{1}, \ldots, \mu_{n}} \beta\right)\left(\delta \supset_{v_{1}, \ldots, v_{m}} \tau\right) . \supset . \alpha \supset_{\mu_{1}, \ldots, \mu_{n}} \beta \\
& \text { 1. }\left(\alpha \supset_{\mu_{1}, \ldots, \mu_{n}} \beta \text {.つ. } \alpha \supset_{\mu_{1}, \ldots, \mu_{n}} \beta\right) .: \text { : . } \\
& \left(\delta \supset v_{1}, \ldots, v_{m} \tau \text {.Ј. } \delta \supset_{v_{1}, \ldots, v_{m}} \tau\right): \supset: \\
& \left(\alpha \supset \mu_{1}, \ldots, \mu_{n} \beta\right)\left(\delta \supset_{v_{1}, \ldots, v_{m}} \tau\right) \text {. } \supset . \alpha \supset_{\mu_{1}, \ldots, \mu_{n}} \beta \quad \operatorname{simp}, \mathrm{DR}(\mathrm{UI}) \\
& \text { 2. } \alpha \supset_{\mu_{1}, \ldots, \mu_{n}} \beta \text {. つ. } \alpha \supset_{\mu_{1}, \ldots, \mu_{n}} \beta \quad \text { Theo }_{(\mathrm{c})} \\
& \text { 3. }\left(\delta \supset v_{1}, \ldots, v_{m} \tau \text {. つ. } \delta \supset_{v_{1} \ldots, v_{m}} \tau\right): \supset \text { : } \\
& \left(\alpha \supset \mu_{1}, \ldots, \mu_{n} \beta\right)\left(\delta \supset_{v_{1}, \ldots, v_{m}} \tau\right) \text {. . } \alpha \supset_{\mu_{1}, \ldots, \mu_{n}} \beta \quad 1,2, \mathrm{Pp}_{4}
\end{aligned}
$$

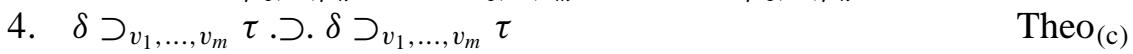

$$
\begin{aligned}
& \text { 5. }\left(\alpha \supset_{\mu_{1}, \ldots, \mu_{n}} \beta\right)\left(\delta \supset_{v_{1}, \ldots, v_{m}} \tau\right) . \supset . \alpha \supset_{\mu_{1}, \ldots, \mu_{n}} \beta \quad \text { 3, 4, } \mathrm{Pp}_{4} \\
& \text { 6. }\left(\alpha \supset_{\mu_{1}, \ldots, \mu_{n}} \beta\right)\left(\delta \supset_{v_{1}, \ldots, v_{m}} \tau\right) . \supset_{x, y, z, w} \cdot \alpha \supset_{\mu_{1}, \ldots, \mu_{n}} \beta \quad \text { 5, UG }
\end{aligned}
$$

It should be clear that similar propositional theses for Russell's other principles are forthcoming as theorem schemata.

In order to avoid having to write out the long clauses of the propositional theses, let us introduce schematic letters ' $p$ ', ' $q$ ', ' $r$ ' which are to refer to well-formed formulas expressing formal or material implications. (These schematic letters must not be confused with Russell's ' $p$ ', ' $q$ ', ' $r$ ', etc. Russell's letters are just individual variables and, accordingly, we replaced them with ' $x$ ', ' $y$ ' , ' $z$ ', etc.) We can now put 


$$
\begin{array}{ll}
\mathrm{id} * & \vdash p \supset p \\
\text { simp* } & \vdash p q . \supset . p \\
\text { syll* } & \vdash p \supset q: \supset: q \supset r . \supset . p \supset r \\
\mathrm{imp} * & \vdash p \cdot \supset . q \supset r: \supset: p q . \supset . r \\
\text { exp* } & \vdash p q . \supset . r: \supset: p \cdot \supset \cdot q \supset r \\
\text { comp* } & \vdash p \supset q: \supset: p \supset r . \supset .(p . \supset . q r) \\
\text { reduc* } & \vdash p \supset q . \supset . p: \supset: p .
\end{array}
$$

The availability of schemata id*, simp*, syll*, imp*, exp*, comp*, and reduc*, validates derived propositional rules. For instance, we have:

DR(syll*) From $p \supset q$ and $q \supset r$, infer $p \supset r$.

DR(imp*) From $p . \supset . q \supset r$, infer $p q . \supset . r$.

DR(exp*) From $p q . \supset . r$, infer $p . \supset . q r$.

$\mathrm{DR}($ comp*) From $p \supset q$ and $p \supset r$, infer $p . \supset$. $q r$.

The justifications are obvious.

Now for each tautology of sentential logic, there are different analogs in the system of Principles. Consider, for example, the tautology,

$$
\text { (1) } A . \rightarrow . B \rightarrow A
$$

(The symbol ' $\rightarrow$ ' here is a statement connective and ' $A$ ' and ' $B$ ' are schematic letters for well-formed formulas.) Russell's system has,

(1a) $p . \supset . q \supset p$.

Call this the "propositional analog" of the sentential tautology. Russell's system also has what are "nonpropositional analogs" of sentential tautologies, however. For our example above there is,

(1b) $x \supset x: \supset_{x, y}: y \supset y . \supset .(x . \supset . y \supset x)$.

Now every instance of (1a) is provable from (1b). But it turns out that the derivation of nonpropositional analogs commonly involves the use of the propositional analogs. So it is important to arrive at propositional analogs first.

With the propositional analogs *id, *simp, *syll, *imp, *exp, *comp, and *reduc at hand, we can show the semantic completeness of Principles with respect to the tautologies of modern sentential logic. In what follows we shall arrive at propositional analogs of the following schemata.

$$
\begin{aligned}
& A . \rightarrow . B \rightarrow A \\
& A . \rightarrow . B \rightarrow C: \rightarrow: A \rightarrow B . \rightarrow . A \rightarrow C \\
& \sim \sim A \rightarrow A
\end{aligned}
$$

Any sentential calculus which takes these axiom schemata, has ' $F$ ' as a primitive well-formed formula (for a necessarily false formula), Modus Ponens as its inference rule, and the definitions

$$
\begin{array}{ll}
\sim A & =_{d f} \quad A \rightarrow F, \\
A \& B & =_{d f} \quad A . \rightarrow . B \rightarrow F: \rightarrow: F \\
A \vee B & =_{d f} \quad A \rightarrow B . \rightarrow B
\end{array}
$$


is known to be semantically complete. ${ }^{12}$ Call this sentential calculus ' $(F)$ '. Our task then, is to prove propositional analogs of the sentential schemata of $(F)$. To demonstrate semantic completeness with respect to tautologies let us begin with the following theorem schemata. Many will validate derived rules, and we shall frequently exploit such derived rules for convenience.

Theorem* ${ }_{1} \quad \vdash p . \supset . q \supset p$

1. $\quad p q \cdot \supset . p$

2. $\quad p q \cdot \supset . p: \supset: p . \supset . q \supset p$

3. $\quad p . \supset . q \supset p$

Theorem*2 $*_{2} \vdash p \supset p p$

1. $\quad p \supset p$

2. $\quad p \supset p: \supset: p \supset p . \supset . p \supset p p$

3. $\quad p \supset p: \supset: p \supset p p$

4. $\quad p \supset p p$ simp*

$\exp ^{*}$

$1,2, \mathrm{Pp}_{4}$

Theorem* $_{3} \quad \vdash(p \supset q) p: \supset: q$

1. $\quad p \supset q \cdot \supset \cdot p \supset q$

$\mathrm{id}^{*}$

2. $(p \supset q) p: \supset: q$

1, DR(imp*)

Theorem* $_{4} \quad \vdash p q . \supset . q$

1. $q \supset q: \supset: p . \supset . q \supset q$

Theo* ${ }_{1}$

2. $\quad p . \supset . q \supset q$

$1, \mathrm{id}^{*}, \mathrm{Pp}_{4}$

3. $p q \cdot \supset . q$

2, DR(imp*)

Theorem* $_{5} \quad \vdash p . \supset . q \supset r: \supset: p \supset q . \supset . p \supset r$

1. $(p . \supset . q \supset r)((p \supset q) p): \supset: p . \supset . q \supset r$

1, simp*

2. $\quad(p . \supset . q \supset r)((p \supset q) p): \supset:(p \supset q) p$

Theo* $_{4}$

3. $\quad(p \supset q) p . \supset . p$

$\mathrm{Theo}_{4}{ }_{4}$

4. $\quad(p . \supset . q \supset r)((p \supset q) p) . \supset . p$

2, 3, DR(syll*)

5. $\quad(p . \supset . q \supset r)((p \supset q) p) . \supset .(p . \supset . q \supset r) p$

$1,4, \mathrm{DR}(\mathrm{comp} *)$

6. $\quad(p . \supset . q \supset r) p . \supset . q \supset r$

Theo* $_{3}$

7. $(p . \supset . q \supset r)((p \supset q) p) . \supset . q \supset r$

$5,6, \mathrm{DR}\left(\right.$ syll $\left.^{*}\right)$

8. $\quad(p \supset q) p \cdot \supset \cdot q$

Theo* $_{3}$

9. $\quad(p . \supset . q \supset r)((p \supset q) p) \supset q$

$2,8, \mathrm{DR}\left(\mathrm{syll}^{*}\right)$

10. $\quad(p . \supset . q \supset r)((p \supset q) p)$.. . $(q \supset r) q$

7, 9, DR(comp*)

11. $\quad(q \supset r) q . \supset . r$

Theo* $_{3}$

12. $\quad(p . \supset . q \supset r)((p \supset q) p) . \supset . r$

10, 11, DR(syll*)

13. $\quad p . \supset . q \supset r: \supset:(p \supset q) p . \supset . r$

$12, \mathrm{DR}\left(\exp ^{*}\right)$

14. $(p \supset q) p . \supset . r: \supset: p \supset q . \supset . p \supset r$ exp*

15. $\quad p . \supset . q \supset r: \supset: p \supset q . \supset . p \supset r$

13, 14, DR(syll*) 
Theorem* $_{6} \quad \vdash p q . \supset . q p$
1. $p q \cdot \supset . p$
simp*
2. $\quad p q . \supset . q$
Theo* 4
3. $p q . \supset . q p$
1, 2, DR(comp*)

Theorem* $_{7}\left(\mathrm{comm}^{*}\right) \quad \vdash p . \supset . q \supset r: \supset: q . \supset . p \supset r$
1. $\quad p . \supset . q \supset r: \supset: p q . \supset . r$
imp*
2. $\quad q p \supset p q . \supset .(p q . \supset . r: \supset: q p$.,$r)$
syll*
3. $q p \supset p q$
Theo* 6
4. $\quad p q \supset r . \supset . q p \supset r$
2, $3, \mathrm{Pp}_{4}$
5. $\quad p . \supset . q \supset r: \supset: q p \supset r$
$1,4, \mathrm{DR}\left(\mathrm{syll}^{*}\right)$
6. $q p \supset r: \supset: q . \supset . p \supset r$
$\exp ^{*}$
7. $\quad p . \supset . q \supset r: \supset: q . \supset . p \supset r$
$5,6, \mathrm{DR}\left(\mathrm{syll}^{*}\right)$

Theorem* ${ }_{8} \quad \vdash z \supset z: \supset_{z}: z \cdot \supset . q \supset z$

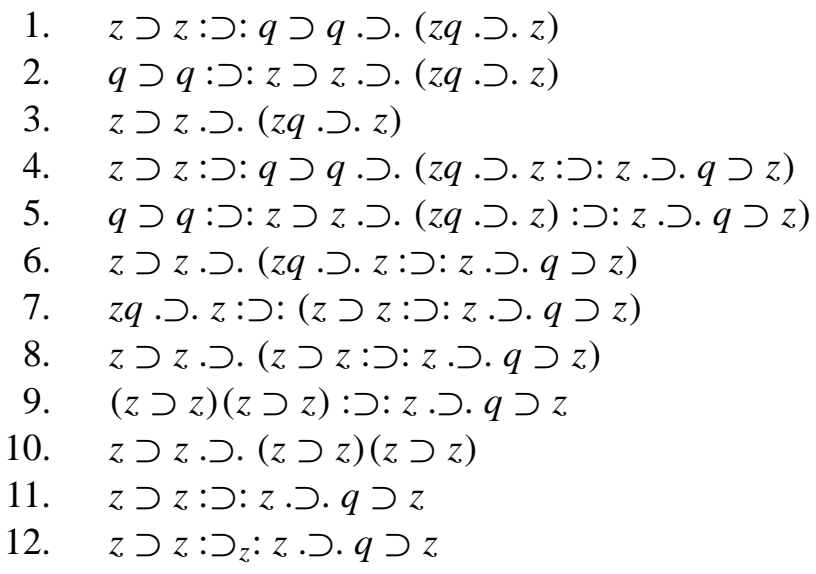

simp, DR(UI)

$1, \mathrm{DR}\left(\mathrm{comm}^{*}\right)$

2, id*, $\mathrm{Pp}_{4}$

exp, DR(UI)

4, DR(comm*)

$5, \mathrm{id}^{*}, \mathrm{Pp}_{4}$

6, DR(comm*)

$3,7, \operatorname{DR}($ syll*)

8, DR(imp*)

Theo* $_{2}$

9, 10, DR(syll*)

$11, \mathrm{UG}$

This is a partly nonpropositional analog of Theo* ${ }_{1}$.

Theorem* $_{9} \quad \vdash f \supset q$

1. $x \supset x . \supset_{x} . x: \supset: q \supset q . \supset . q$

2. $\quad q \supset q . \supset .\left(x \supset x . \supset_{x} . x: \supset: q\right)$

Theo $_{(\mathrm{a})}$

3. $\quad q \supset q$

4. $x \supset x . \supset_{x} . x: \supset: q$

$1, \mathrm{DR}\left(\mathrm{comm}^{*}\right)$

5. $f \supset q$

2, 3, $\mathrm{Pp}_{4}$

4, $d f(f)$

Theorem* ${ }_{10} \quad \vdash--p \supset p$

1. $\quad p \supset f . \supset . p: \supset: p$

2. $\quad p \supset f . \supset . f: \supset: f \supset p . \supset .(p \supset f . \supset . p)$

reduc* syll* 


$$
\begin{array}{llr}
\text { 3. } & f \supset p: \supset:(p \supset f . \supset . f) . \supset .(p \supset f . \supset . p) & 2, \mathrm{DR}\left(\mathrm{comm}^{*}\right) \\
\text { 4. } & p \supset f . \supset . f: \supset: p \supset f . \supset . p & 3, \mathrm{Theo}^{*}, \mathrm{Pp}_{4} \\
\text { 5. } & p \supset f . \supset . f: \supset: p & 1,4, \mathrm{DR}_{\left(\mathrm{syll}^{*}\right)} \\
\text { 6. } & --p \supset p & 5, d f(-)
\end{array}
$$

It might at first be thought that the presence of Theo* ${ }_{1}$, Theo* ${ }_{5}$, and Theo* ${ }_{10}$ are enough to show semantic completeness, but this is not so. We need to prove the equivalence of Russell's negation with ours and the equivalence of Russell's conjunction with ours.

Theorem* $^{*}{ }_{11} \vdash \neg p \supset-p$

$$
\begin{array}{llr}
\text { 1. } & z \supset z \cdot \supset_{z} p \supset z: \supset: f \supset f . \supset . p \supset f & \text { Theo }_{(a)} \\
\text { 2. } & f \supset f . \supset .(z \supset z \cdot \supset z \cdot p \supset z: \supset: p \supset f) & 1, \mathrm{DR}\left(\mathrm{comm}^{*}\right) \\
\text { 3. } & f \supset f & \text { id }^{*} \\
\text { 4. } & z \supset z \cdot \supset_{z} p \supset z: \supset: p \supset f & 2,3, \mathrm{Pp}_{4} \\
\text { 5. } & \neg p \supset-p & 4, d f(\neg), d f(-)
\end{array}
$$

Theorem* ${ }_{12} \quad \vdash p: \supset: z \supset z . \supset . z . \supset_{z}: z \supset z . \supset . p \supset z$ where $z$ is not free in $p$.

$$
\begin{aligned}
& \text { 1. } \quad z \supset z . \supset . z \supset z: \supset: z \supset z . \supset \text {. } \\
& {[(p: \supset: z \supset z . \supset . z) \supset(p(z \supset z) \supset z)] \quad \text { imp, DR(UI) }} \\
& \text { Abbreviate, putting ' } m \text { ' for ' } z \supset z \text { ' } \\
& \text { 2. } \quad m \supset m: \supset: m . \supset .[(p . \supset . m \supset z) \supset(p m \supset z)] \quad 1, d f(m) \\
& \text { 3. } m \supset m \\
& \text { 4. } \quad m \supset[(p . \supset . m \supset z) \supset(p m \supset z)] \\
& \text { 5. } \quad m(p . \supset . m \supset z) . \supset . p m \supset z \\
& \text { 6. } \quad m p \supset p m \\
& \text { 7. } 6: \supset: p m \supset z . \supset . m p \supset z \\
& \text { 8. } \quad p m \supset z \cdot \supset . m p \supset z \\
& \text { 9. } \quad m(p . \supset . m \supset z) . \supset . m p \supset z \\
& \text { 10. } \quad m \supset m: \supset: p \supset p . \supset .[m p \supset z: \supset: m . \supset . p \supset z) \\
& \text { 11. } m p \supset z: \supset: m . \supset . p \supset z \\
& \text { 12. } \quad m(p . \supset . m \supset z): \supset: m . \supset . p \supset z \\
& \text { 13. } \quad m \supset[m(p . \supset . m \supset z) . \supset . p \supset z] \\
& \text { 14. }[m(p . \supset . m \supset z) . \supset . p \supset z] \supset \\
& \text { [m:つ: }(p . \supset . m \supset z) . \supset . p \supset z] \\
& \text { 15. } \quad m \supset[m: \supset:(p . \supset . m \supset z) . \supset . p \supset z] \\
& \text { 16. } m m: \supset:(p . \supset . m \supset z) . \supset . p \supset z \\
& \text { 17. } m \supset \mathrm{mm} \\
& \text { 18. } \quad m: \supset:(p . \supset . m \supset z) . \supset . p \supset z \\
& \text { 19. } \quad p . \supset . m \supset z: \supset: m . \supset . p z
\end{aligned}
$$

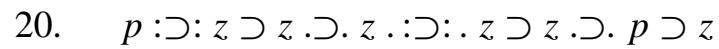

$$
\begin{aligned}
& \text { 21. } \quad p: \supset: z \supset z \cdot \supset . z \cdot \supset_{z}: z \supset z \cdot \supset . p \supset z \\
& 6,7, \mathrm{Pp}_{4} \\
& 5,8, \mathrm{DR}\left(\mathrm{syll}^{*}\right) \\
& \exp , \mathrm{DR}(\mathrm{UI}) \\
& 10, \mathrm{id}^{*}, \mathrm{Pp}_{4} \\
& \text { 20, UG }
\end{aligned}
$$

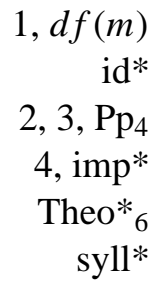


Theorem* ${ }_{13} \quad \vdash-p \supset \neg p$
1. $\quad p \supset f: \supset:(f: \supset: z \supset z . \supset . z) . \supset$.
$(p: \supset: z \supset z . \supset . z)$
syll*
2. $(f: \supset: z \supset z . \supset . z): \supset: p \supset f . \supset$.
$(p: \supset: z \supset z . \supset . z)$
1, DR(comm*)
3. $f: \supset: z \supset z \cdot \supset . z$
4. $\quad p \supset f . \supset .(p: \supset: z \supset z . \supset . z)$
$\mathrm{Theo}_{(\mathrm{a})}, d f(f)$
2, $3, \mathrm{Pp}_{4}$

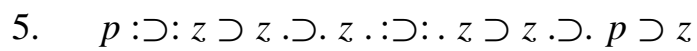
, DR(UI)
6. $\quad p \supset f: \supset: z \supset z \cdot \supset . p \supset z$
$4,5, \mathrm{DR}\left(\right.$ syll*) $^{*}$
7. $\quad p \supset f: \supset_{z}: z \supset z \cdot \supset . p \supset z$
6, UG
8. $\quad p \supset f: \supset: z \supset z \cdot \supset_{z} \cdot p \supset z$
7, Theo $_{(\mathrm{b})}, \mathrm{Pp}_{4}$
9. $-p \supset \neg p$
$8, d f(-), d f(\neg)$

Theorem* ${ }_{14} \vdash z \supset z \cdot \supset_{z} \cdot f \supset z$

1. $\quad f: \supset: z \supset z . \supset . z$

2. $\quad 1: \supset: z \supset z . \supset . f \supset z$

Theo $_{(\mathrm{a})}, d f(f)$

3. $\quad z \supset z \cdot \supset \cdot f \supset z$

Theo* ${ }_{12}$, DR(UI)

4. $\quad z \supset z \cdot \supset_{z} \cdot f \supset z$

$1,2, \mathrm{Pp}_{4}$

$3, \mathrm{UG}$

Theorem* ${ }_{15} \vdash z \supset q: \supset_{z}:-z \supset q . \supset . q$, where $z$ is not free in $q$.
1. $z \supset q: \supset:-q \supset-z$
2. $\quad-q \supset-z: \supset:-z \supset q . \supset .-q \supset q$
syll*, $d f(-)$ syll*
3. $z \supset q: \supset:-z \supset q . \supset .-q \supset q$
$1,2, \operatorname{DR}\left(\operatorname{syll}^{*}\right)$
4. $\quad-q \supset q . \supset . q$
reduc*, $d f(f)$
5. $\quad-z \supset q . \supset .-q \supset q: \supset:(-q \supset q . \supset . q) \supset$ $(-z \supset q \cdot \supset . q)$
syll*
6. $\quad-q \supset q . \supset \cdot q: \supset:(-z \supset q . \supset .-q \supset q) \supset$ $(-z \supset q \cdot \supset . q)$
5, DR(comm*)
7. $\quad-z \supset q . \supset .-q \supset q: \supset:-z \supset q$.,$q$
$4,6, \mathrm{Pp}_{4}$
8. $\quad z q: \supset:-z \supset q \cdot \supset . q$
$3,7, \mathrm{DR}\left(\right.$ syll* $\left.^{*}\right)$
9. $\quad z \supset q: \supset_{z}-z \supset q . \supset . q$
$8, \mathrm{UG}$

Theorem $_{16} \quad \vdash x y \cdot \supset_{x, y} \cdot x \& y$
1. $\quad\left(z \supset z . \supset_{z}: x \cdot \supset . y \supset z: \supset: z\right) \supset$
$(f \supset f . \supset: x . \supset . y \supset f: \supset: f)$
Theo $_{(a)}$
2. $f \supset f: \supset: x y . \supset . x \& y$
$1, d f\left(\mathrm{comm}^{*}\right), d f(x y), d f(\&)$
3. $x y . \supset . x \& y$
4. $x y . \supset_{x, y} x \& y$
$2, \mathrm{id}^{*}, \mathrm{Pp}_{4}$
3, UG

Theorem* ${ }_{17} \vdash p q$.Ј. $p \& q$

Immediate from Theo 16 , by DR(UI). 
Theorem* $_{18} \quad \vdash p \& q$.Ј. $p q$

$$
\begin{aligned}
& \text { 1. } \quad q \supset z: \supset: z \supset f . \supset . q \supset f \\
& \text { 2. } \quad z \supset f: \supset: q \supset z . \supset . q \supset f \\
& \text { 3. } \quad(p . \supset . q \supset z) . \supset .(q \supset z . \supset . q \supset f) \supset \\
& (p . \supset . q \supset f) \\
& \text { 4. } \quad(q \supset z . \supset . q \supset f) . \supset .(p . \supset . q \supset z) \supset \\
& (p . \supset . q \supset f) \quad 3, \mathrm{DR}(\mathrm{comm} *) \\
& \text { 5. } \quad z \supset f . \supset .(p . \supset . q \supset z) \supset(p . \supset . q \supset f) \quad \text { 2, 4, DR(syll*) } \\
& \text { 6. } \quad(p . \supset . q \supset z) \supset(p . \supset . q \supset f): \supset \text { : } \\
& (p . \supset . q \supset f: \supset: z) \supset(p . \supset . q \supset z: \supset: z) \quad \text { syll* } \\
& \text { 7. } \quad z \supset f . \supset .(p . \supset . q \supset f: \supset: z) \supset
\end{aligned}
$$

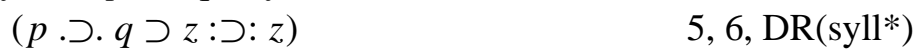

$$
\begin{aligned}
& \text { 8. ( } \quad(p . \supset . q f: \supset: z): \supset: \\
& z \supset f . \supset .(p . \supset . q \supset z: \supset: z) \quad \text { 7, DR(comm*) } \\
& \text { 9. ( } \quad(\supset . \neg \supset f: \supset: f): \supset: \\
& f \supset z . \supset .(p . \supset . q \supset f: \supset: z) \quad \text { syll* } \\
& \text { 10. } \quad(p . \supset . q \supset f: \supset: f)(f \subset z) \supset(p . \supset . q \supset f: \supset: z) \quad \text { 9, imp* } \\
& \text { 11. } \quad(p \& q)(f \supset z): \supset: z \supset f . \supset \text {. } \\
& (p . \supset . q \supset z: \supset: z) \\
& 8,10, \mathrm{DR}\left(\operatorname{syll}^{*}\right), d f(\&) \\
& \text { 12. } \quad(f \supset z)(p \& q) \cdot \supset .(p \& q)(f \supset z) \\
& \text { 13. } \quad(f \supset z)(p \& q): \supset: z \supset f . \supset \text {. } \\
& (p . \supset . q \supset z: \supset: z) \\
& \text { Theo* }{ }_{6}
\end{aligned}
$$

14. $f \supset z . \supset$.

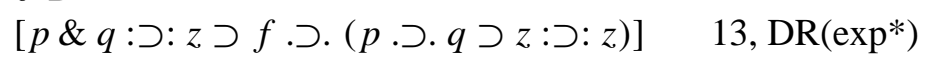

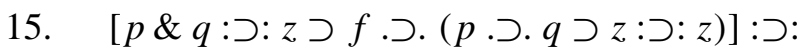

16. $f \supset z: \supset: z \supset f . \supset$.

$$
z \supset f . \supset .[p \& q \supset(p . \supset . q \supset z: \supset: z)] \quad \text { comm* }
$$

$$
\text { [p\&q.つ. ( } p . \supset . q \supset z: \supset: z)]
$$

17. $z \supset z \cdot \supset . f \supset z$

Theo* ${ }_{14}, \mathrm{DR}(\mathrm{UI})$

18. $\quad z \supset z: \supset: z \supset f . \supset$.

$$
\begin{aligned}
& \text { [p\&q.つ. ( } p . \supset . q \supset z: \supset: z)] \\
& \text { 16, 17, DR(syll*) }
\end{aligned}
$$

Now let us call ' $p . \supset . q \supset z$ ' by the name ' $w$ ' for convenience.

$$
\begin{aligned}
& \text { 19. } \quad z \supset z: \supset: z \supset f . \supset .[p \& q . \supset . w \supset z] \\
& \text { 20. } \quad z \supset z: \supset: z \cdot \supset . w \supset z \\
& \text { 21. } \quad w \supset z: \supset: p \& q . \supset . w \supset z \\
& \text { 22. } \quad z . \supset . w \supset z: \supset: 21 \text {. } w \text {. } \\
& (z: \supset: p \& q . \supset . w \supset z) \quad \text { syll* } \\
& \text { 23. } 21: \supset:(z . \supset . w \supset z) \supset \\
& (z: \supset: p \& q . \supset . w \supset z) \quad 22, \mathrm{DR}\left(\mathrm{comm}^{*}\right) \\
& \text { 24. } \quad z . \supset . w \supset z: \supset:(z: \supset: p \& q . \supset . w \supset z) \\
& \text { 25. } z \supset z \cdot \supset .(z: \supset: p \& q \cdot \supset . w \supset z)
\end{aligned}
$$


Finally, abbreviate, putting ' $s$ ' for ' $p \& q . \supset . w \supset z$.'
26. $z \supset z: \supset: z \supset f . \supset . s$
27. $z \supset z: \supset: z \supset s$
28. $\quad z \supset z: \supset:(z \supset s)(z \supset f . \supset . s)$
29. $(z \supset s)(z \supset f . \supset . s): \supset$ :
$z \supset s . \supset .(z \supset f . \supset . s)$
30. $z \supset s . \supset .(z \supset f . \supset . s): \supset: s$
31. $z \supset z . \supset . s$
$19, d f(s)$
$25, d f(s)$
$19, d f(s)$
$25, d f(s)$
26, 27, DR(comp*)
32. $\quad z \supset z . \supset .(p \& q . \supset . w \supset z)$
$\exp ^{*}$
$\mathrm{Theo}_{15}$, DR(UI), $d f(-)$
33. $z \supset z . \supset .[p \& q: \supset:(p . \supset . q \supset z: \supset: z)]$
28, 29, 30, DR(syll*)
34. $\quad p \& q . \supset .[z \supset z . \supset .(p . \supset . q \supset z: \supset: z)]$
$31, d f(s)$
$32, d f(w)$
35. $\quad p \& q . \supset_{z} \cdot[z \supset z . \supset .(p . \supset . q \supset z: \supset: z)]$
33, $\mathrm{DR}\left(\mathrm{comm}^{*}\right)$
36. $\quad p \& q . \supset .\left[z \supset z \cdot \supset_{z} \cdot(p \cdot \supset \cdot q \supset z: \supset: z)\right]$
37. $p \& q \cdot \supset . p q$
34, UG
35, Theo*(b), $\mathrm{Pp}_{4}$
36, $d f(p q)$

Our theorems show that the system of Principles is complete with respect to analogs of sentential tautologies.

5 Independence and reduction In Principles Russell wondered whether Reduction was independent (10], p. 17). In discussion of this paper with me, Byrd suggested a proof that Reduction is independent as follows.

Consider the subsystem $\left(\mathrm{P}^{*}\right)$ of $(\mathrm{P})$ which consists of the propositional axiom schemata id*, syll*, simp*, imp*, exp*, and comp* together with $\mathrm{Pp}_{4}$. With Russell's definition of tilde and the conjunction sign, the schemata $\mathrm{id}^{*}$, simp* $^{*}$, syll* $^{*}, \mathrm{imp}^{*}$, exp*, comp* are intuitionistically valid, but reduc* is not. On the basis of Byrd's argument then, we can show that Reduction is independent. We have lately seen that reduc* is not an intuitionistic semantic consequence of the others. Now the subsystem $\left(\mathrm{P}^{*}-\right)$, which is just $\left(\mathrm{P}^{*}\right)$ with reduc* removed, is intuitionistically sound (in the sense that any thesis of the system is a propositional analog of a sentential tautology which is intuitionistically valid). So reduc*, being a propositional analog of a sentential tautology that is not intuitionistically valid, is not a thesis of the subsystem $\left(\mathrm{P}^{*}-\right)$. Consider then the subsystem $(\mathrm{P}-)$ which is just $(\mathrm{P})$ with axiom Reduction removed. Every thesis of $(\mathrm{P}-)$ that is a propositional analog of a sentential tautology is a thesis of the subsystem $\left(\mathrm{P}^{*}-\right)$. It follows that reduc* is not a theorem schema of $(\mathrm{P}-)$. We now have our result. For if Reduction were a thesis of $(\mathrm{P}-)$, then reduc* would be a theorem schema of $(\mathrm{P}-)$ for it is immediate from Reduction. Accordingly, Reduction is not a thesis of $(\mathrm{P}-)$. Reduction is independent in $(\mathrm{P})$.

Curiously, a rather innocuous looking change in the system of Principles $(\mathrm{P})$ alters the independence result for reduc*. If we replace Russell's ' $x y$ ' with our ' $x \& y$ ' in Russell's axioms simp, imp, exp, and comp, then reduc* is provable after all. Call the system with the change "Principles+" $(\mathrm{P}+)$. We now prove: ${ }^{13}$

$$
\begin{array}{ccc}
\text { neg* } & \vdash_{p+}--p \supset p & \\
& & \\
\text { 1. } & (p . \supset . p \supset f) \& p . \supset . p \supset f & \text { Theo* }_{3} \\
\text { 2. } & (p . \supset . p \supset f) \& p . \supset . p & \text { Theo* }_{4}
\end{array}
$$




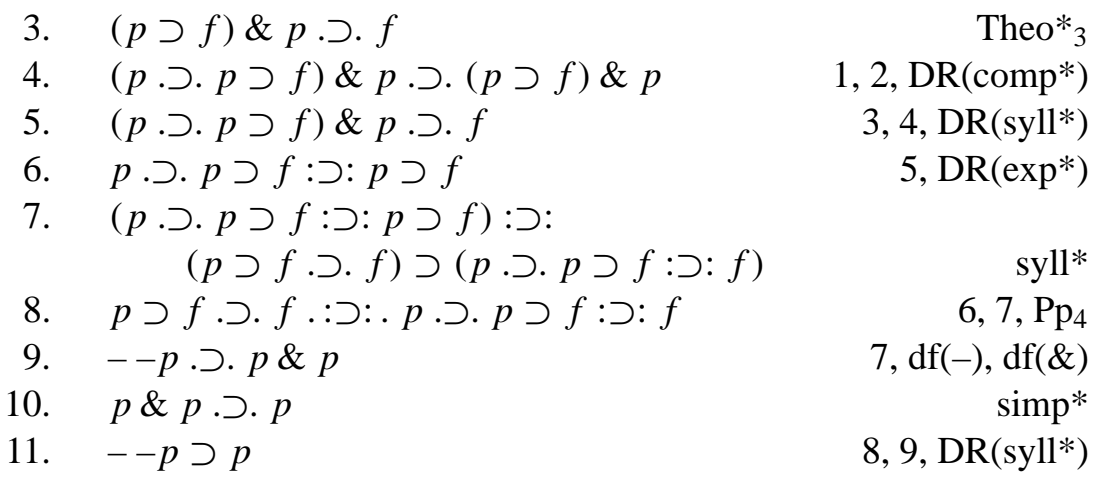

It follows that $(\mathrm{P}+)$ is semantically complete. So reduc* + is provable in $(\mathrm{P}+)$.

If we can prove Reduction in $(\mathrm{P}+)$ then, since we have the equivalences of Russell's negation and ours, Russell's conjunction and ours, the systems are deductively equivalent - that is, every thesis of $(\mathrm{P})$ is a thesis of $(\mathrm{P}+)$, and conversely. In the next section, we shall find that in $(\mathrm{P})$ and $(\mathrm{P}+)$ we can transform any propositional ana$\log$ of a sentential tautology into a nonpropositional analog. So we shall arrive at the non-independence Reduction in $(\mathrm{P}+)$.

In his 1905 manuscript "On Fundamentals," Russell seems aware that a change in his definition of the conjunction sign achieves the non-independence of reduc* (and Reduction) ([13], p. 413). In considering the matter of the independence of Russell's principles, it is worth noting that Vuillemin 17 observes that id* is provable from syll*, simp*, exp*, reduc*. The proof is obvious:

$$
\begin{array}{ll}
\text { 1. } & p(\neg p \supset p) \supset p \\
\text { 2. } & p: \supset: \neg p \supset p \cdot \supset p \\
\text { 3. } & \neg p \supset p \cdot \supset p: \supset: p \\
\text { 4. } & p \supset p
\end{array}
$$

$$
\begin{array}{r}
\text { simp* } \\
1, \mathrm{DR}\left(\exp ^{*}\right) \\
\text { reduc* }^{*} \\
2,3, \mathrm{DR}\left(\text { syll* }^{*}\right)
\end{array}
$$

One must not, however, think that $\mathrm{Pp}_{1}$ (and so id*) are not independent. Taking a rule of uniform replacement, Vuillemin proceeds as if the principles were axioms of a modern sentential calculus. This forgets that Russell's $\mathrm{Pp}_{1}$ is required to derive propositional analogs in the first place. The principle $\mathrm{Pp}_{1}$ is indispensable to the original system of Principles (and Principles + ) and *id is its immediate consequence. Vuillemin also cites Guillaume's proof of comp* from id*, syll*, imp*, exp* and reduc*. Guillaume's proof does not suffer from the same flaw as Vuillemin's proof of *id. One can proceed without Composition to id*, syll*, imp*, exp*, and reduc*, and then produce Guillaume's proof of comp*. Since our next section shows that one can arrive at nonpropositional analogs from the propositional, it follows that Composition is not independent in $(\mathrm{P})$.

\section{Completeness with respect to nonpropositional analogs of sentential tautologies} How can we know that Principles is semantically complete with respect to nonpropositional analogs of sentential tautologies? We can know this by showing that it is possible to transform any propositional analog into a nonpropositional one. The fol- 
lowing can be proved by meta-linguistic induction on the number of connectives in a well-formed formula. ${ }^{14}$

Theorem schema $(\mathrm{d})$

$\vdash$ If $\ulcorner A v\urcorner$ is any context of $v$ built up solely from $\supset$, then $\mu \supset \mu: \supset_{\mu}$ : $A[\{\mu \vee \mu\} \mid v] . \equiv . A[\mu \mid v]$, with $\mu$ free for $v$ in $A$.

Here I put: $\alpha \equiv \beta={ }_{d f}(\alpha \supset \beta)(\beta \supset \alpha)$. For the base case, $\ulcorner A v\urcorner$ must be one among:
(a) $v \supset v$
(b) $v \supset x$
(c) $x \supset v$

In situation (a) we have to show

Theorem $_{19} \vdash \mu \supset \mu . \supset_{\mu} \cdot(\mu \vee \mu . \supset . \mu \vee \mu: \equiv: \mu \supset \mu)$.

1. $\quad \mu \supset \mu: \supset:(\mu \vee \mu . \supset . \mu \vee \mu) . \supset . \mu \supset \mu$ Theo* ${ }_{1}$

2. $(\mu \supset \mu)(\mu \supset \mu): \supset: \mu \supset \mu . \supset . \mu \supset \mu$ $\log$

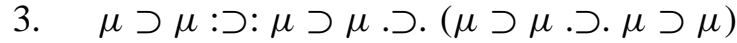

2, DR(exp*), $\mathrm{Pp}_{4}$

4. $\quad \mu \supset \mu: \supset:(\mu \vee \mu . \supset . \mu \vee \mu: \supset: \mu \vee \mu \supset$ $(\mu \supset \mu: \supset: \mu \vee \mu . \supset . \mu \vee \mu)$ $1,3, \mathrm{DR}(\mathrm{comp} *)$

5. $\quad \mu \supset \mu . \supset .(\mu \vee \mu . \supset . \mu \vee \mu: \equiv: \mu \vee \mu)$

$4, d f(\equiv)$

6. $\mu \supset \mu . \supset_{\mu} .(\mu \vee \mu . \supset . \mu \vee \mu: \equiv: \mu \vee \mu)$

$5, \mathrm{UG}$

For situations (b) and (c) we shall need:

Theorem $_{20} \quad \vdash \mu \supset \mu: \supset_{\mu}: \mu . \supset . \mu \vee \mu$

1. $\quad \mu \supset \mu . \supset .(\mu: \supset: \mu \supset \mu . \supset . \mu)$

Theo* ${ }_{8}$, DR(UI)

2. $\mu \supset \mu: \supset: \mu . \supset . \mu \vee \mu$

$1, d f(v)$

3. $\mu \supset \mu: \supset_{\mu}: \mu . \supset . \mu \vee \mu$

Theorem $_{21} \quad \vdash \mu \supset \mu: \supset_{\mu}: \mu \vee \mu . \supset . \mu$

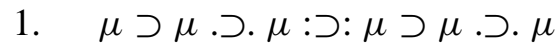

$\mathrm{id}^{*}$

2. $\mu \vee \mu: \supset: \mu \supset \mu$. $\quad \mu$

3. $\mu \supset \mu: \supset: \mu \vee \mu . \supset . \mu$

4. $\mu \supset \mu: \supset_{\mu}: \mu \vee \mu . \supset . \mu$

2, Theo* ${ }_{12}, \mathrm{DR}(\mathrm{UI}), \mathrm{Pp}_{4}$

$3, \mathrm{UG}$

We now have situation (b):

Theorem $_{22} \quad \vdash \mu \supset \mu . \supset \mu, x .(\mu \vee \mu . \supset . x: \equiv: \mu \supset x)$

1. $\mu \supset \mu: \supset: \mu . \supset . \mu \vee \mu$

$\mathrm{TheO}_{20}, \mathrm{DR}(\mathrm{UI})$

2. $\quad \mu . \supset . \mu \vee \mu: \supset:(\mu \vee \mu . \supset . x) . \supset . \mu \supset x$

syll*

3. $\mu \supset \mu . \supset .(\mu \vee \mu . \supset . x: \supset: \mu \supset x)$

4. $\mu \supset \mu: \supset: \mu \vee \mu . \supset . \mu$ 
5. $(\mu \vee \mu . \supset . \mu): \supset: \mu \supset x . \supset .(\mu \vee \mu$.つ. $x)$

6. $\quad \mu \supset \mu: \supset: \mu \supset x . \supset .(\mu \vee \mu . \supset . x)$

7. $\mu \supset \mu . \supset .(\mu \vee \mu . \supset . x: \supset: \mu \supset x)$ $(\mu \supset x: \supset: \mu \vee \mu . \supset . x)$

8. $\quad \mu \supset \mu . \supset .(\mu \vee \mu . \supset . x: \equiv: \mu \supset x)$

9. $\quad \mu \supset \mu . \supset \mu, x .(\mu \vee \mu . \supset . x: \equiv: \mu \supset x)$

\author{
syll* \\ 4, 5, DR(syll*) \\ 3, 6, DR(comp*) \\ $7, d f(\equiv)$
}

Situation (c) is akin to situation (b) and left for the reader. This completes the base.

For the inductive step, assume that

$$
\mu \supset \mu . \supset_{\mu} \cdot A[\mu \vee \mu \mid v]: \equiv: A[\mu \mid v],
$$

for all $\ulcorner A v\urcorner$ with fewer than $n$ connectives. We want to show it holds for $\ulcorner A v\urcorner$ with $n$ connectives. Now $\ulcorner A v\urcorner$ is $\ulcorner B v \supset C v\urcorner$, where $\ulcorner B v\urcorner$ and $\ulcorner C v\urcorner$ have fewer than $n$ connectives. Accordingly, by the inductive hypothesis, we have:

$$
\begin{aligned}
& \mu \supset \mu: \supset_{\mu}: B[\mu \vee \mu \mid v] \equiv . \equiv[\mu \mid v], \\
& \mu \supset \mu: \supset_{\mu}: C[\mu \vee \mu \mid v] . \equiv . C[\mu \mid v] .
\end{aligned}
$$

From these the reader can easily see that it is straightforward to demonstrate that we have

$$
\mu \supset \mu: \supset \mu: A[\mu \vee \mu \mid v]: \equiv: A[\mu \mid v],
$$

for $\ulcorner A v\urcorner$ with $n$ connectives.

The purpose of the above schema is to enable a transformation from any quantifier-free propositional analog to a nonpropositional analog. To illustrate, let us transform an instance of reduc* to arrive at Reduction.

$\vdash \quad x \supset x: \supset_{x, y}: y \supset y . \supset .[x \supset y . \supset . x: \supset: x]$

0. $\quad(x \vee x) \supset(y \vee y) . \supset .(x \vee x): \supset:(x \vee x) \quad$ reduc*

1. $\quad x \supset x . \supset .[(x \vee x) \supset(y \vee y) . \supset .(x \vee x): \supset:$

$$
(x \vee x)] \supset[x \supset(y \vee y) . \supset . x: \supset: x] \quad \text { Theo }_{(\mathrm{d})}, \log
$$

2. $[(x \vee x) \supset(y \vee y) . \supset .(x \vee x): \supset:(x \vee x)]: \supset:$

$$
x \supset x . \supset .[x \supset(y \vee y) . \supset . x: \supset: x] \quad 1, \mathrm{DR}\left(\mathrm{comm}^{*}\right)
$$

3. $x \supset x . \supset .[x \supset(y \vee y) . \supset . x: \supset: x] \quad 0,2, \mathrm{Pp}_{4}$

4. $\quad y \supset y . \supset .\{x \supset x . \supset .[x \supset(y \vee y) . \supset . x: \supset: x]\}$

$$
\{x \supset x . \supset .[x \supset y . \supset . x: \supset: x]\} \quad \text { Theo }_{(\mathrm{d})}, \log
$$

5. $\{x \supset x . \supset .[x \supset(y \vee y) . \supset . x: \supset: x]\}: \supset:$

$$
y \supset y . \supset .\{x \supset x . \supset .[x \supset y . \supset . x: \supset: x]\} \quad 4, \mathrm{DR}\left(\mathrm{comm}^{*}\right)
$$

6. $\quad y \supset y . \supset .\{x \supset x . \supset .[x \supset y . \supset . x: \supset: x]\}$

7. $\quad x \supset x: \supset: y \supset y . \supset .[x \supset y . \supset . x: \supset: x]$

$3,5, \mathrm{Pp}_{4}$

8. $\quad x \supset x: \supset_{x, y}: y \supset y . \supset .[x \supset y . \supset . x: \supset: x]$

It should be clear that we can always transform in this way. The above schema validates the following schema for $(\mathrm{P})$ : 
Theorem Schema $(\mathrm{e})$

$\vdash B[\{z \supset w\} \mid v] . \supset_{z, w} . C[\{z \supset w\} \mid v]: \supset: \mu \supset \mu . \supset_{\mu} . B[\mu \mid v] \supset$ $C[\mu \mid v]$, where $\ulcorner B[\{z \supset w\} \mid v] \supset C[\{z \supset w\} \mid v]\urcorner$ is a propositional analog of a sentential tautology.

(To state this meta-theorem, we have to use a conditional form because quantifiers always index a conditional sign. We intend that there might be cases where $v$ does not occur in $B$ and/or $v$ does not occur in $C$.) This schema shows the semantic completeness of $(\mathrm{P})$ with respect to nonpropositional analogs of the sentential tautologies.

7 Odds and ends With the semantic completeness of (P) with respect to analogs of sentential tautologies, it follows that our system $(\mathrm{P}+)$ is deductively equivalent with $(\mathrm{P})$. We have lately seen that in $(\mathrm{P})$ we can transform any propositional analog of a sentential tautology into a nonpropositional analog. The transformation holds in $(\mathrm{P}+)$ as well. Accordingly, the above demonstration of Reduction from reduc* in $(\mathrm{P})$ holds as well in (P+). The equivalence of our conjunction with Russell's and our tilde with Russell's holds in $(\mathrm{P}+)$. Accordingly, $(\mathrm{P})$ and $(\mathrm{P}+)$ are deductively equivalent.

We know as well that $(\mathrm{P}+)$ and therefore $(\mathrm{P})$, are consistent. For this we need only apply a version of the common consistency proof for the predicate calculus. Eliminate all definitions, except for that of "f", change all occurrences of " $f$ " to " $F$ ", then delete all quantifier indices, change all individual variables to sentential letters, and change all occurrences of " $\supset$ " to “ $\rightarrow$ ". Every axiom of $(\mathrm{P}+)$ is now a tautology. Russell's $\mathrm{Pp}_{4}$ becomes the transformation rule Modus Ponens, which preserves tautologyhood, and Russell's Rule (CP) preserves it as well. So any inconsistency in $(\mathrm{P}+)$ would show up in the sentential calculus $(\mathrm{F})$ above. Since $(\mathrm{F})$ is known to be consistent, so is $(\mathrm{P}+)$. Since $(\mathrm{P})$ is deductively equivalent with $(\mathrm{P}+)$, we know that $(\mathrm{P})$ is consistent.

What follows are some odds and ends. We have some interesting theses of the system $\mathrm{P}$ that are not analogs (propositional or nonpropositional) of sentential tautologies.

Theorem $_{24} \vdash y \supset y: \supset_{x, y} . x \supset f . \supset . x \supset y$

$$
\begin{array}{ll}
\text { 1. } & y \supset y . \supset . f \supset y \\
\text { 2. } & x \supset f: \supset: f \supset y . \supset . x \supset y \\
\text { 3. } & f \supset y: \supset: x \supset f . \supset . x \supset y \\
\text { 4. } & y \supset y: \supset: x \supset f . \supset . x \supset y \\
\text { 5. } & y \supset y: \supset x, y: x \supset f . \supset . x \supset y
\end{array}
$$

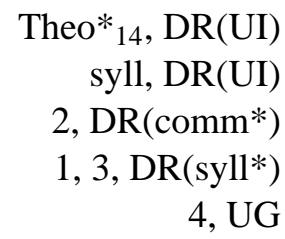

The next theorem shows that the proposition ' $--x$ ' does not imply $x$ when $x$ is not a proposition.

$$
\begin{aligned}
& \text { Theorem }_{25} \quad \vdash-(x \supset x) . \supset_{x} \cdot-(--x . \supset . x) \\
& \text { 1. }--x \supset x: \supset: x \supset x \\
& \text { 2. } \quad x \supset x . \supset . f . \supset: .--x \supset x . \supset . f
\end{aligned}
$$

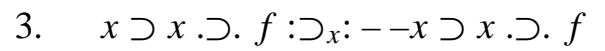

$$
\begin{aligned}
& \mathrm{Pp}_{2}, \mathrm{UI} \\
& \text { 4. }-(x \supset x) \cdot \supset_{x} \cdot-(--x \supset x) \\
& \text { 1, syll*, } \mathrm{Pp}_{4} \\
& \text { 2, UG } \\
& 3, d f(-)
\end{aligned}
$$


With Russell's definition of the disjunction sign, we get:

Theorem $_{26} \quad \vdash-(y \supset y): \supset_{x, y}:-(x \vee y)$

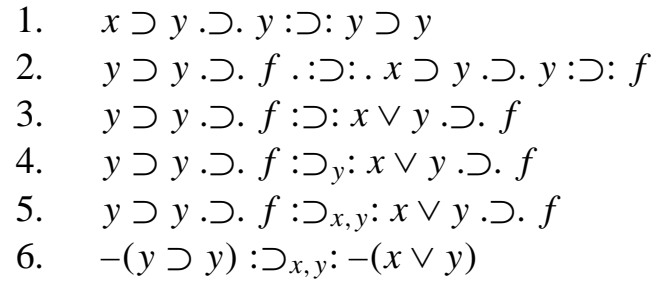

$\mathrm{Pp}_{2}, \mathrm{DR}(\mathrm{UI})$

1, syll*$^{*}, \mathrm{Pp}_{4}$

2, $d f(\vee)$

3, UG

4, UG

$5, d f(-)$

That is, $y$ 's being a proposition implies, for all $x$ and $y, x \vee y$.

Theorem $_{27} \vdash y \supset y . \supset_{x, y} . .-(x \supset x) . \supset . x \vee y^{15}$

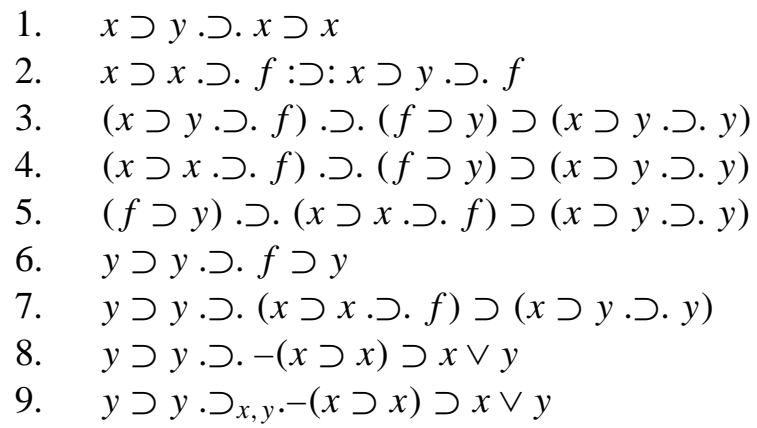

$\mathrm{Pp}_{2}, \mathrm{DR}(\mathrm{UI})$

1, syll*, $\mathrm{Pp}_{4}$

2, syll*

$2,3, \mathrm{DR}(\mathrm{syll} *)$

4, DR(comm*)

Theo* ${ }_{14}, \mathrm{DR}(\mathrm{UI})$

$5,6, \mathrm{DR}(\mathrm{syll} *)$

$7, d f(-), d f(\vee)$

$8, \mathrm{UG}$

This says that $y$ 's being a proposition and $x$ 's not being a proposition, implies for all $x$ and $y, x \vee y$. There is a similar result concerning conjunction:

Theorem $_{28} \quad \vdash-(x \supset x): \supset_{x, y}: x \& y$

$$
\begin{array}{ll}
\text { 1. } & x . \supset . y \supset f: \supset: x \supset x \\
\text { 2. } & x \supset x . \supset . f: \supset: x \cdot \supset . y \supset f: \supset: f \\
\text { 3. } & x \supset x . \supset . f: \supset: x \& y \\
\text { 4. } & x \supset x . \supset . f: \supset_{y}: x \& y \\
\text { 5. } & x \supset x . \supset . f: \supset_{x, y}: x \& y \\
\text { 6. } & -(x \supset x) \cdot \supset_{x, y} \cdot x \& y
\end{array}
$$

From the equivalence in (P) of Russell's conjunction and our \&, we see that $x$ 's not being a proposition implies $\{x y\}$. On the other hand, we have:

Theorem $_{29} \quad \vdash-(y \supset y) . \supset_{x, y} . x \& y: \supset: y \supset_{y} y$

1. (y つ y.つ. $\left.f: \supset_{x, y} x \& y\right) \supset(y \supset y . \supset . f: \supset: f \& y) \quad$ Theo $_{(a)}$

2. $\quad\left(y \supset y . \supset . f: \supset_{x, y} x \& y\right) \supset$

$$
(y \supset y . \supset . f: \supset:[f . \supset . y \supset f: \supset: f]) \quad 1, d f(\&)
$$

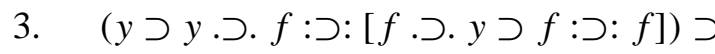

$$
f . \supset . y \supset f: \supset: y \supset y
$$




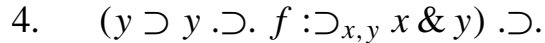

$$
(f \text {. } \supset \text {. } y \supset f: \supset: y \supset y)
$$$$
2,3, \mathrm{DR}\left(\mathrm{syll}^{*}\right)
$$

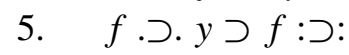

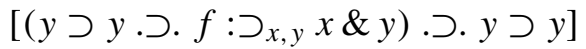$$
\text { 4, DR(comm*) }
$$$$
\text { Theo* }{ }_{9}
$$$$
\text { 6. } f . \supset . y \supset f
$$$$
5,6, \mathrm{Pp}_{4}
$$

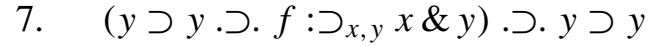

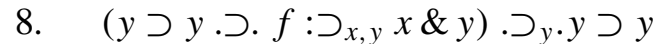$$
\text { 7, UG }
$$

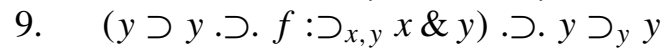$$
\text { 10. } \quad-(y \supset y) . \supset_{x, y} . x \& y: \supset: y \supset_{y} y
$$

Curiously, Byrd expected a theorem that $\{x y\}$ is implied by $y$ 's being a proposition ([1], p. 351). We can see from Theo 29 that if Byrd were correct, it would follow that every entity is a proposition. Now every thesis of the system is either a propositional analog of a sentential tautology or is a conditional (material or formal) whose antecedent is of the form

$$
\ulcorner\mu \supset \mu\urcorner .
$$

Accordingly, there is no thesis of the system of the form

$$
\left\ulcorner\mu \supset_{\mu} \mu\right\urcorner \text {. }
$$

That is, it is not a theorem of $(\mathrm{P})$ or $(\mathrm{P}+)$ that every entity is a proposition. This is certainly as it should be. And it follows that there is no theorem of the system which says that $y$ 's not being a proposition implies $\{x y\}$.

\section{NOTES}

1. See Frege 44], p. 371 and Russell 414], p. 62.

2. As we now know from the work of Tarski and Gödel this is indeed the case.

3. In fact, 1906 finds Russell embracing the fact that 'designates-in-s' cannot be a predicate of $s$, and using this to reply to the Richard paradox. See [12], p. 209.

4. He did reject Peano's conclusion that any progression has equal claim to being regarded as the natural numbers. Logicism's logical analyis of cardinal number reveals the real natural numbers in Russell's view.

5. See Russell [10], p. 7, and also Russell [12, p. 205.

6. Quine ([9], pp. 31, 257, 268) has precisely the same view and rejects predicate variables. But surely no one would accuse Quine of rejecting the meta-language/object-langauge distinction.

7. See [10], p. 15; and Whitehead and Russell [18], p. 115.

8. Actually, Principles did not adopt any notation here. The work offered an analysis of the constituents of propositions, that are formal implications, by employing a theory of denoting concepts. The details of this theory and its analysis of the constituents of propositions expressed by nominalized well-formed formulas containing single letters used as variables need not detain us here. 
9. In reconstructing the calculus of Principles, Church 3 has special propositional variables, and employs the statement connective ' $\rightarrow$ '. Then, noticing the oddity of $\ulcorner p \supset$ $q .=. r\urcorner$, he adds a special quadruple bar to express identity where propositions are concerned. Once we see that Principles has only individual variables and ' $\supset$ ', there is no reason for the quadruple bar. We have ' $\{x \supset y\}=z$ ' as a well-formed formula.

10. We should not be misled by the occurrence of 'truth' in *7.11, as if this shows a conflation of theory and meta-theory. The rule UG is meta-linguistic, and as such it would be perfectly admissible to speak of well-formed formulas of a form as being true.

11. Byrd 1 calls attention to this passage as well.

12. See Church [2], p 72.

13. The theorem schemata used below refer to those proved above with Russell's definition of the conjunction sign. But so long as Russell's axiom Simplification has been adjusted, these proofs can easily be rewritten with our definition of ' $\&$ '.

14. Since we have completeness, the following proofs avail themselves of some commonplace inferences of sentential logic, annotating with 'log'.

15. This was pointed out in Byrd [1, p. 351.

\section{REFERENCES}

[1] Byrd, M., "Russell, logicism and the choice of the logical constants," Notre Dame Journal of Formal Logic, vol. 30 (1989), pp. 343-361.Zbl 0685.03005IMR 91a:03001 3 . 3,17,17,17

[2] Church, A., Introduction to Mathematical Logic, Princeton University Press, Princeton, 1956. Zbl 0073.24301|MR 18:631a 7

[3] Church, A., "Russell's theory of the identity of propositions," Philosophia Naturalis, vol. 21 (1984), pp. 515-522. MR 87h:03001 7

[4] Frege, G., "On Herr Peano's Begriffsschrift and my own," Australasian Journal of Philosophy, vol. 47 (1969), pp. 1-14. 1.12.7.

[5] Hylton, P., Russell, Idealism and the Emergence of Analytic Philosophy, Oxford University Press, Cambridge, 1990. 1.11.1.1

[6] McGuiness, B., Gottlob Frege: Philosophical and Mathematical Correspondence, University of Chicago Press, Chicago, 1980. ZZbl 0502.03002|MR 81d:03001 3

[7] Peano, G., (ed.), Formulaire de Mathèmatiques, Bocca, Turin, 1889. 2,2,2

[8] Peano, G., "Arithmetices Principia," Novo Methodo Exposita, Turin, 1889. Reprinted in van Heijenoort, From Frege to Gödel, Cambridge, 1967, pp. 83-97. Zbl 01748963 MR 2003a:03008 2

[9] Quine, W.V.O., Set Theory and Its Logic, Harvard University Press, Harvard, 1963. Zbl 0193.30402|MR 43:37 7

[10] Russell, B., The Principles of Mathematics, W.W. Norton \& Company, New York, 1996.

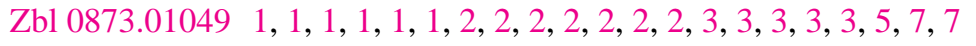

[11] Russell, B., "The Theory of Implication," American Journal of Mathematics, vol. 28, 1906, pp. 159-202. 
[12] Russell, B., "On 'Insolubilia' and their solution by symbolic logic," pp. 90-214 in Essays in Analysis, edited by D. Lackey, George Braziller, New York, 1973. 7.7

[13] Russell, B., "On fundamentals," pp. 359-413 in The Collected Papers of Bertrand Russell, vol. 4, edited by A. Urquhart and A. Lewis, Routledge, London, 1994. 5

[14] Russell, B., "Mathematics and the Metaphysicians," pp. 59-74 in Mysticism and Logic, Longmans, New York, 1963. 7

[15] van Heijenoort, J., "Logic as language and logic as calculus," Synthese, vol. 17 (1967), pp. 324-330. Zbl 0154.00305 1

[16] van Heijenoort, J., "Systéme et Mètasystéme ches Russell," pp. 111-122 in Logic Colloquium 85, edited by Paris Logic Group, North-Holland, Amsterdam, 1987. Zbl 0638.03002|MR 88g:03002 1

[17] Vuillemin, J., Lecons sur la Premiére Philosophie de Russell, Colin, Paris, 1968. 3.5

[18] Whitehead, A. N., and B. Russell, Principia Mathematica vol. 1, Cambridge University Press, Cambridge, 1910. Zb1 0877.01042 MR 2000c:03007 1.77

Department of Philosophy

University of Iowa

269 English Philosophy Building

Iowa City, Iowa 52242-1408

email: gregory-landini@uiowa.edu 